\title{
Modulation of sleep by trafficking of lipids through the Drosophila blood brain barrier
}

Gregory Artiushin, Fu Li, Amita Sehgal*

Howard Hughes Medical Institute and Chronobiology and Sleep Institute, Perelman School of Medicine, University of Pennsylvania, Philadelphia, Perelman School of Medicine, University of Pennsylvania, Philadelphia

${ }^{*}$ Corresponding author 


\begin{abstract}
Endocytosis through Drosophila glia is a significant determinant of sleep amount and occurs preferentially during sleep in glia of the blood brain barrier (BBB). To identify metabolites whose trafficking is mediated by sleep-dependent endocytosis, we conducted metabolomic analysis of flies that have increased sleep due to a block in glial endocytosis. We report that acylcarnitines, molecules that conjugate with long chain fatty acids to promote their transport, accumulate in heads of these animals. In parallel, to identify transporters and receptors whose loss contributes to the sleep phenotype caused by blocked endocytosis, we screened genes enriched in barrier glia for effects on sleep. We find that knockdown of lipid transporters LRP1\&2 as well as carnitine transporters ORCT1\&2 increases sleep. In support of the idea that the block in endocytosis affects trafficking through specific transporters, knockdown of LRP or ORCT transporters also increases acylcarnitines in heads. We propose that lipid species, such as acylcarnitines, are trafficked through the BBB via sleep-dependent endocytosis, and their accumulation in the brain increases the need for sleep.
\end{abstract}

\title{
Introduction
}

Although the regulation of sleep is normally studied on a behavioral and circuit level, there is increasing evidence for a role of basic cellular physiology. For instance, we found that disruption of endocytic and trafficking pathways in glia increases sleep in Drosophila (Artiushin, Zhang et al. 2018). Glia of the Drosophila blood/hemolymph brain barrier (BBB) emerged as a new cellular locus of sleep regulation in this study, such that genetically manipulating endocytosis in these cells alone was sufficient to increase sleep. As the increased sleep appeared to reflect higher sleep need, we asked if sleep is typically required for endocytosis through the BBB and found that this was indeed the case. However, the nature of the molecules trafficked through the BBB in a sleep-dependent manner was not known.

Barriers that separate the solutes of blood/hemolymph of the periphery from the interstitial fluid of the central nervous system display a rich profile of transporters, receptors, and trafficking proteins, which often reflect their unique functions. The Drosophila barrier glia populations share many conserved features with vertebrate barriers which employ endothelial and astrocytic populations (DeSalvo, Hindle et al. 2014, Weiler, Volkenhoff et al. 2017). For instance, both are capable of moving lipids and carbohydrates, ions, amino 
acids and xenobiotics (Weiler, Volkenhoff et al. 2017). Furthermore, the fly barrier populations may serve specialized roles in metabolism, as not only the conduit of energy sources from the periphery, but also by containing the enzymatic machinery necessary for processing energy sources (Volkenhoff, Weiler et al. 2015), and secreting signals in reference to nutritional state (Chell and Brand 2010, Speder and Brand 2014).

Given that much of the traffic through the barrier involves energetic substrates, we conducted metabolomic profiling to identify candidate metabolites whose trafficking may be inhibited by an endocytosis block in glia. To complement this approach, we asked if specific glial proteins mediate this trafficking. As genetic manipulations to block endocytosis can directly impact endocytosis-dependent carrier traffic as well as indirectly affect levels of membrane-associated transporters/receptors by altered recycling, we performed a knockdown screen to broadly search for barrier genes involved in sleep regulation. We report here that specific lipid and carnitine transporters act in barrier glia to affect sleep, and that disrupting expression of these transporters or of endocytosis leads to an accumulation of acylcarnitines in the head.

\section{Results}

\section{Expression of Shi in glia induces acylcarnitine accumulation in fly heads}

Our previous work showed that expression of shibire (shi), a dominant negative dynamin that blocks endocytosis, in all or BBB glia increases sleep. To attain an unbiased, global assessment of metabolites that may be relevant to the increased sleep seen in Repo>20xShi.ts1 flies (hereafter referred to as Repo>Shi ${ }^{1}$ ), we conducted LC-MS analysis. Heads of male and female Repo-Gal4 > Shi' flies as well as Gal4 and UAS controls were collected on dry ice with sieves and immediately frozen at $-80{ }^{\circ} \mathrm{C}$. Each sample contained 200 fly heads (equal male and female), with a total of 5 samples per genetic condition.

As an initial analysis, raw signal was scaled per each metabolite in reference to other samples within the dataset, and comparisons were made between Repo>Shi flies and each control, as well as controls to each other by Welch's t-test (Supplementary Table 1). 


\begin{tabular}{|c|c|c|c|c|}
\hline Sub pathway & Biochemical name & $\frac{\text { Gal4 }>U A S}{\text { Gal4Ctrl }}$ & $\frac{\text { Gal4 >UAS }}{U A S C t r l}$ & $\frac{\text { UASCtrl }}{\text { Gal4Ctrl }}$ \\
\hline \multirow{15}{*}{$\begin{array}{l}\text { Fatty Acid } \\
\text { Metabolism } \\
\text { (Acylcarnitine) }\end{array}$} & acetylcarnitine (C2) & 4.25 & 2.68 & \\
\hline & myristoylcarnitine (C14) & 28.81 & 26.39 & 1.09 \\
\hline & palmitoylcarnitine (C16) & 4.52 & 1.74 & 2.60 \\
\hline & palmitoleoycarnitine (C16:1) & 26.50 & 10.35 & 2.56 \\
\hline & stearoylcarnitine (C18) & 1.43 & 1.06 & 1.34 \\
\hline & linoleoylcarnitine (C18:2) & 6.77 & 3.14 & 2.15 \\
\hline & oleoylcarnitine (C18:1) & 4.00 & 1.92 & 2.08 \\
\hline & arachidoylcarnitine (C20) & 1.28 & 1.17 & 1.09 \\
\hline & behenoylcarnitine (C22) & 1.31 & 1.09 & 1.20 \\
\hline & eicosenoylcarnitine (C20:1) & 2.28 & 1.24 & 1.84 \\
\hline & lignoceroylcarnitine (C24) & 0.72 & 0.83 & 0.87 \\
\hline & margaroylcarnitine (C17) & 4.05 & & 2.76 \\
\hline & nervonoylcarnitine (C24:1) & & 1.26 & 1.87 \\
\hline & cerotoylcartinine (C26) & 0.85 & 0.97 & 0.87 \\
\hline & ximenoylcarnitine (C26:1) & 0.75 & 0.88 & 0.85 \\
\hline
\end{tabular}

Table 1. Fatty acid acylcarnitine accumulation in Repos Shi' fly heads

All samples from Repo-GAL4>UAS-Shi', and both parental controls. Welch's t-test was performed on scaled signal for each metabolite, comparing the conditions shown. Green highlighting marks a significant difference $(p \leq 0.05)$ between the groups, where metabolite ratio is $<1.00$, while light green is not significant, but close to the threshold $(0.05<p<0.10)$. Red highlighting marks a significant difference $(p \leq 0.05)$ between groups where metabolite ratio is $\geq 1.00$, and light red is not significant, but close to the threshold $(0.05<p<0.10)$.

Metabolites of interest were those for which signal from the experimental samples was significantly different, in the same direction, when compared to both controls, while controls compared to each were not significant. Of secondary interest were metabolites where a difference was seen in controls, but was proportionally smaller than consistent differences of each control to the experimental samples.

In surveying this dataset, the outstanding functional category, which contained multiple metabolites whose signal was consistently different in experimental animals versus controls, were the acyl-carnitines (Table 1). Furthermore, the fold changes for given metabolites in this group, which consists of fatty acids conjugated to carnitine, were the highest overall. Carnitinylation occurs on fatty acids of various chain lengths, but only a subset of chain lengths in this dataset had sufficient signal, therefore we statistically compared Repo>Shi' flies to both parental controls for metabolites that had signal in at least three of five biological replicates for each genotype. Expression of Shi' in glia increased abundance in fly heads of the following acylcarnitine species: C2, C16, C16*1, C17, C18:1, C18:2* (Figure 1A). The only metabolite of 
this group with less signal in experimental animals was the longer-chain, C24* (Figure 1B). Carnitine and deoxycarnitine were not significantly altered as compared to both controls.
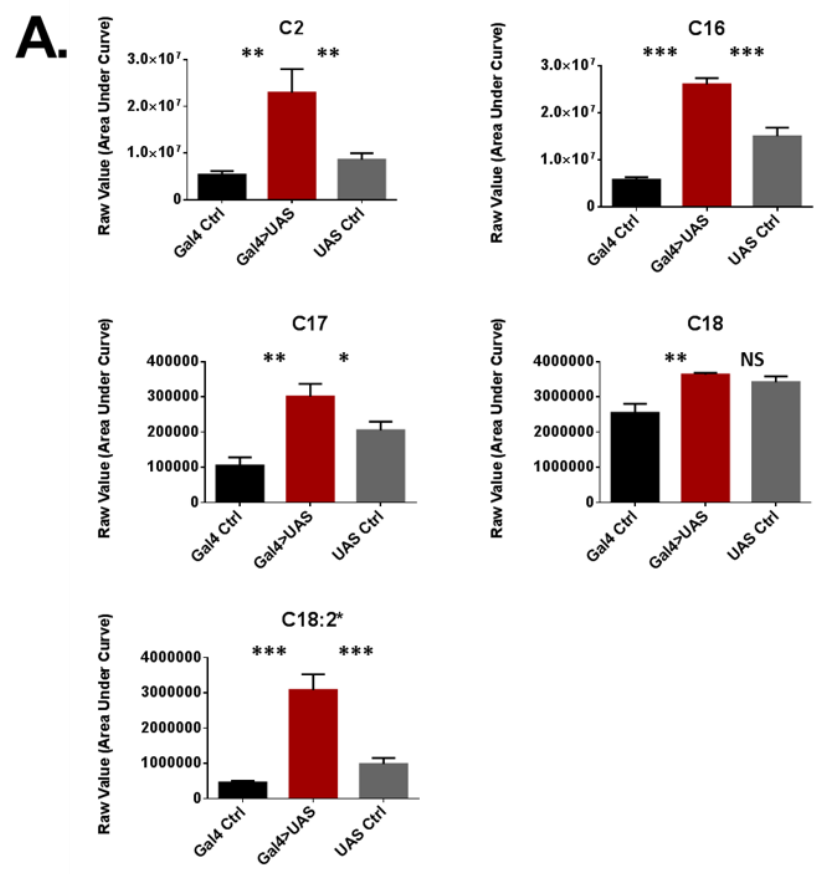

B.
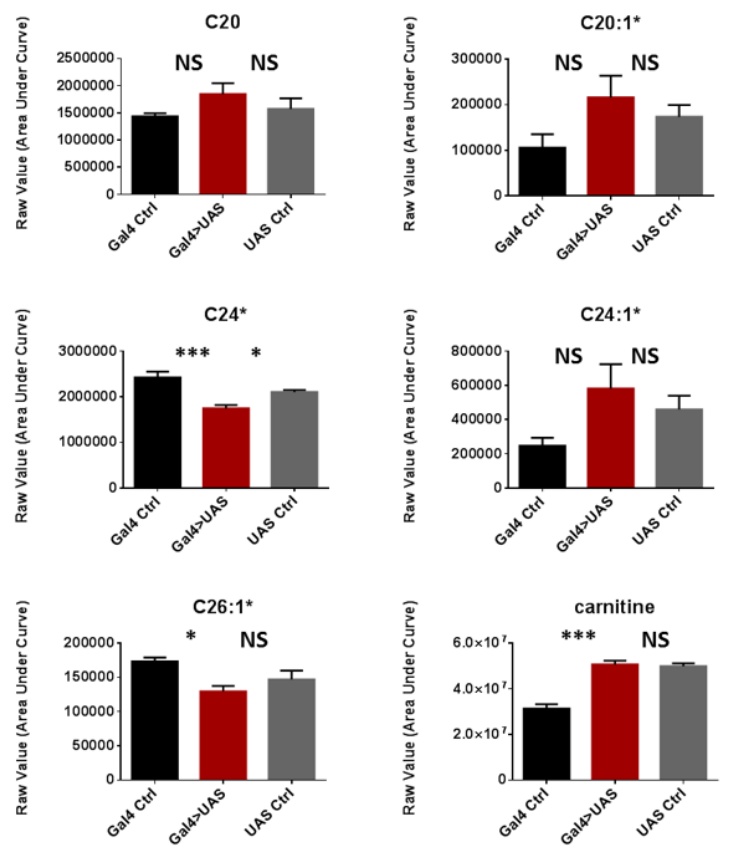
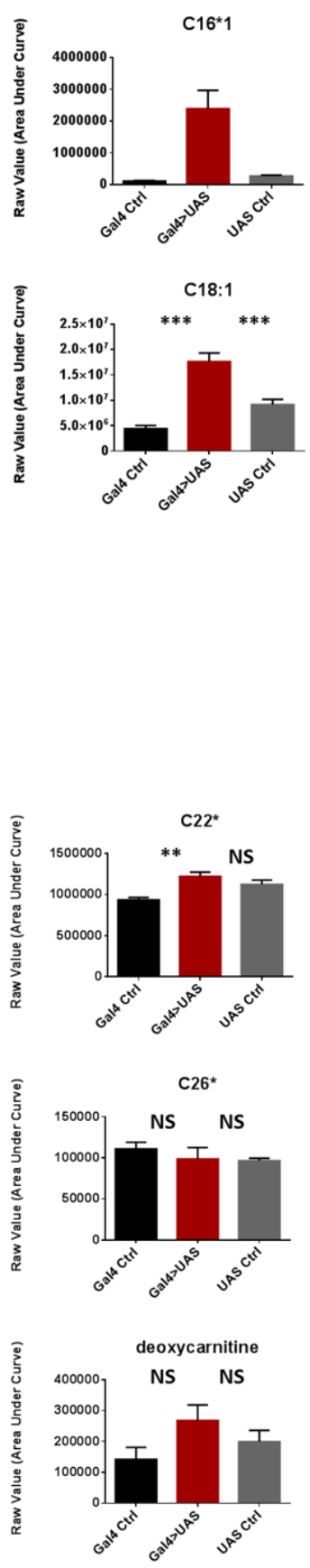

Figure 1: Acylcarnitine levels are increased in Repo $>$ Shi ${ }^{1}$ fly heads

(A) Short and medium chain length or (B) long chain length acylcarnitines from Repo-G4 $>$ UAS-Shi ${ }^{1}$ fly heads and parental controls. The raw signal from LC/MS is plotted, $n=3-5$ samples, of 200 fly heads each. One-way ANOVA, 
with Holk-Sidak post-hoc comparisons. ${ }^{*} p<0.05,{ }^{* *} p<0.01,{ }^{* * *} p<0.001$. Error bars represent standard error of the mean (SEM).

\section{Identification of barrier glia genes that affect sleep}

In addition to identifying metabolites that accumulate as a result of blocked glial endocytosis, we sought to identify glial molecules whose function might be impacted by the block in endocytosis and thereby contribute to the effect on sleep. As noted above, glia of the BBB are the most relevant glial subtype for sleep-dependent endocytosis. We identified genes enriched in barrier glia by referring to transcriptional profiling that compared expression in the two glial populations that comprise the Drosophila BBBsubperineurial and perineurial glia (SPG + PG) - to all neurons, and all glia (DeSalvo, Hindle et al. 2014). Preference was given to previously studied genes, particularly transporters, receptors and those involved in trafficking, although many genes among the top-50 highly expressed in the barrier glia populations were also tested for effects on sleep. Of the genes enriched in barrier glia, we focused on those that showed low variability in expression from sample to sample. UAS-RNAi constructs for candidate genes were expressed with Repo/RepoGS Gal4 drivers and sleep in these lines was compared with that in Gal4 and UAS alone controls. Knockdown of most genes did not produce a significant phenotype, but sleep was increased with knockdown of some transporter genes CG3036, CG6126, mnd, VMAT, CG6836, Rh50, CG4462 (Figure 2- figure supplement 1A), cytoskeleton/trafficking factors CG8036, Vha16, nuf, (Figure 2-figure supplement 1C) as well as Isd-2, acon and MtnA (Figure 2- figure supplement 1D). Meanwhile, knockdown of the transporter gene CG16700 (Supp Figure 2A), cytochrome P450 gene Cyp6a20 (Figure

2- figure supplement 1D) and trafficking factor $C / n 7$ decreased total sleep (Figure 2- figure supplement 1C).

Since the candidate genes were selected based on enrichment within the barrier glia, we chose to examine and secondarily validate promising phenotypes through knockdown with more limited, barrier glia drivers. Thus, we screened a sub-set of the genes suggested by results of the pan-glial screen (Figure 2-figure supplement 1) with drivers that target PG or SPG glia. Knockdown of MtnA (105011 KK), CG6386 (108502 KK), CG4462 (105566 KK), or Isd-2 (102269 KK) did not significantly alter sleep when expressed in either of the barrier glial populations alone (data not shown). Reduction of cyp6a20 in the PG population inconsistently reproduced the pan-glial sleep loss phenotype, so this was not pursued further (data not 
shown). The VMAT gene produces two isoforms, one of which is thought to be specific to glia (RomeroCalderon, Uhlenbrock et al. 2008). Knockdown of VMAT (TRiP HMC02346) in the perineurial glia increased total sleep (Figure 2A), but in the subperineurial glia it had no effect (data not shown), which is consistent with protein expression, as the VMAT-B antibody specifically marks the perineurial glia (DeSalvo et al., 2014). Pan-glial knockdown of neuronal ceroid lipofuscinosis 7 ( Cln 7$)$, which is expressed in the perineurial glia (Mohammed et al., 2017), decreased total sleep (Figure 2C). However, expression of Cln7 RNAi in the PG increased total sleep time, with no significant change produced by expression in the SPG (Figure 2B, C).

A.

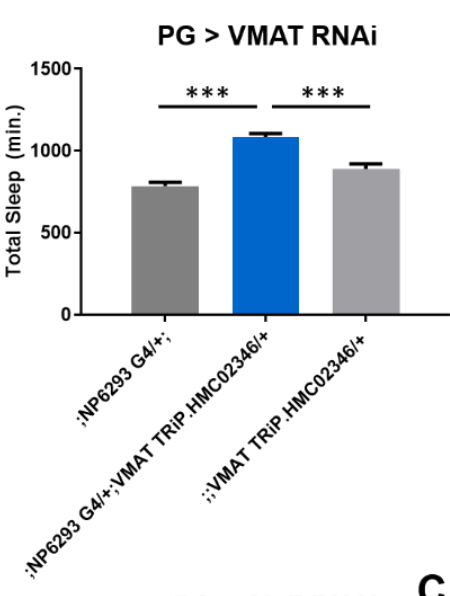

B.

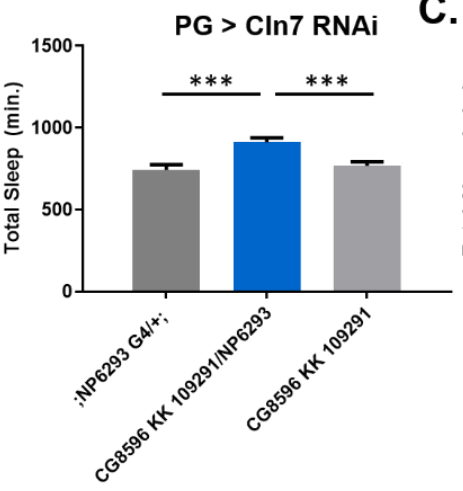

C.

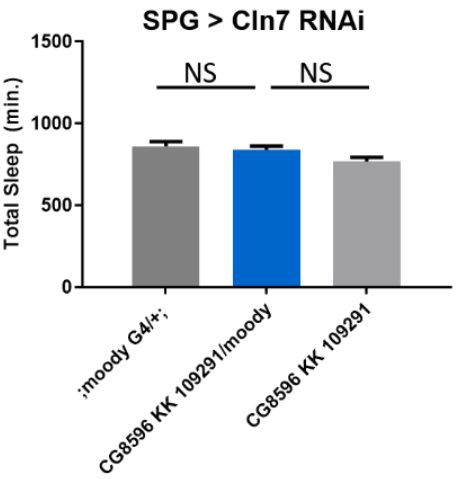

Figure 2: Knockdown of specific barrier-enriched genes with barrier glial drivers

Total sleep in female flies with knockdown of (A) VMAT, UAS- HMC02346 driven by (PG) NP6293-Gal4. n=15-16 per genotype. (B and C) Cln7 (CG8896), UAS-109291 KK driven by (PG) NP6293-Gal4 or (SPG) moody-Gal4. $\mathrm{n}=15-16$ per genotype. One-way ANOVA, with Holk-Sidak post-hoc comparisons. ${ }^{*} p<0.05,{ }^{* \star} p<0.01,{ }^{* \star *} p<0.001$. Error bars represent standard error of the mean (SEM).

\section{Simultaneous knockdown of Lrp1 and Megalin in barrier glia increases sleep}


Although the candidate screen identified barrier genes whose knockdown increases sleep, as does blocking endocytosis in barrier glia, these genes were not obviously linked to the metabolite profile seen with a block in glial endocytosis. The results of metabolomic screening showed changes in lipid, and particularly carnitine-lipid, trafficking. Therefore, we reassessed our screen candidates to consider transporters and receptors which may function in these pathways and could have been missed due to redundancy/lethality. Lrp1 and Megalin (Lrp2) are two LDL receptor-related protein members involved in the transport of lipid carrier proteins at the fly barrier (Brankatschk, Dunst et al. 2014). Expression is likewise found in mammals at the endothelial barrier (Herz 2003).

A.

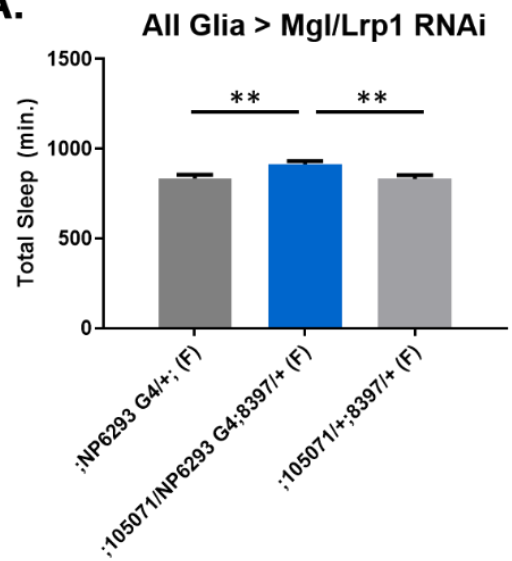

D.

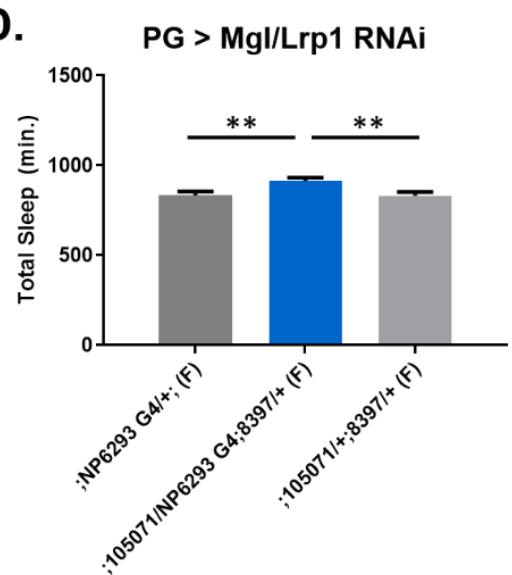

B.

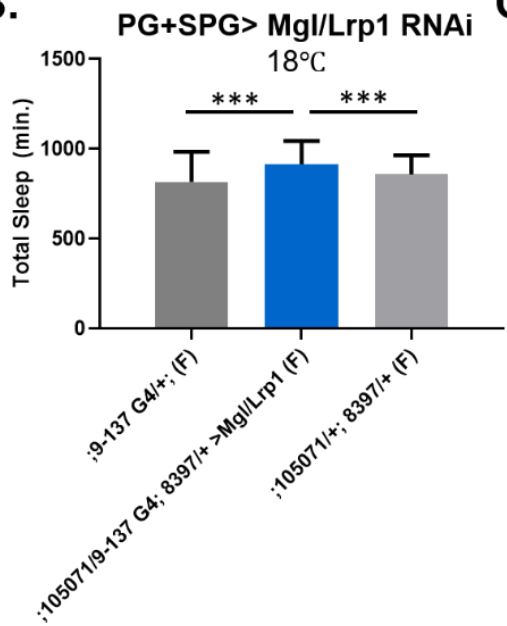

E.

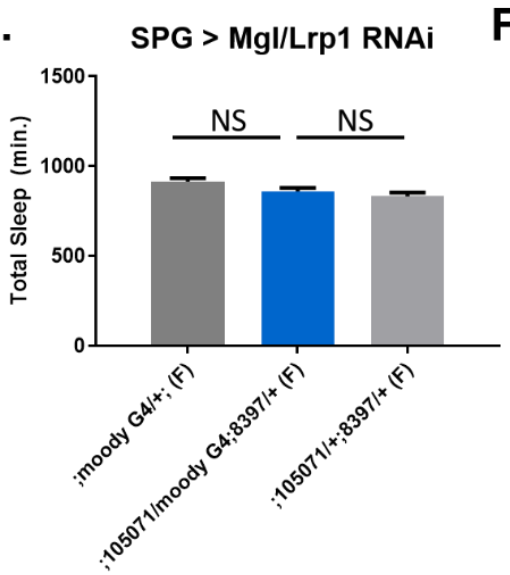

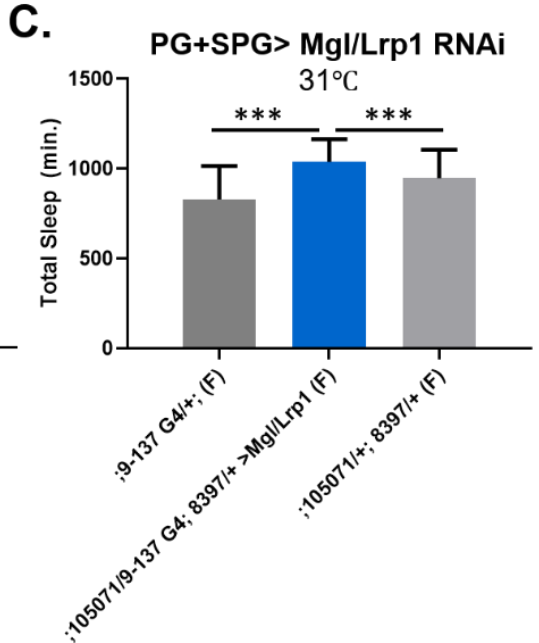

F.

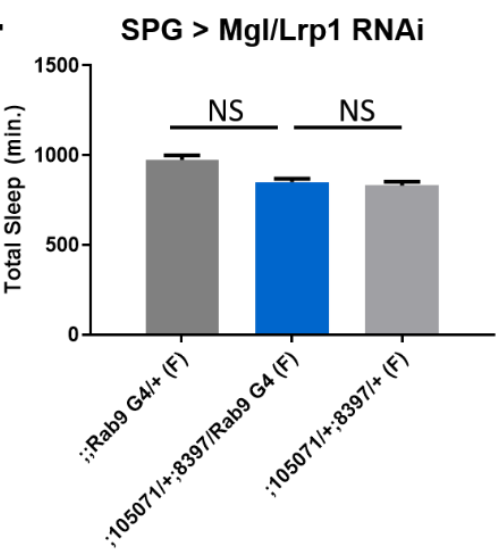

Figure 3. Sleep time changes with knockdown of Lrp genes in all glia or barrier glia

Total sleep in female flies with knockdown of (A) Lrp1 (8397 GD) and Megalin (105071 KK) RNAi driven by Repo-GAL4. $\mathrm{n}=11-16$ per genotype; (B) Lrp1 (8397 GD) and Megalin (105071 KK) RNAi driven by 9-137-GAL4 (PG and SPG) with TubGal80 ts at the permissive temperature of $18^{\circ} \mathrm{C}, \mathrm{n}=30$ - 32 per genotype; (C) $\operatorname{Lrp} 1$ (8397 GD) and Megalin 
(105071 KK) RNAi driven by 9-137-GAL4 (SPG and PG) with TubGal80ts at the restrictive temperature of $31{ }^{\circ} \mathrm{C}, \mathrm{n}=30-$ 32 per genotype.; (D, E, F) Lrp1 (8397 GD) and Megalin (105071 KK) RNAi expressed by: (D) NP6293- Gal4 (PG), n = 13-16 per genotype; (E) moody-GAL4 (SPG), n=16 per genotype; (F) Rab9-GAL4 (SPG), n=13-16 per genotype. One-way ANOVA, with Holk-Sidak post-hoc comparisons. ${ }^{*} p<0.05$, ${ }^{* *} p<0.01$. Error bars represent standard error of the mean (SEM).

Knocking down Lrp1 and Megalin (Lrp2) individually in the pan-glial screen did not significantly alter total sleep time (Figure 2- figure supplement 1A). However, both Lrp1 and Megalin (Lrp2) as well as Orct and Orct2 have been considered to be complementary (Eraly and Nigam 2002, Brankatschk, Dunst et al. 2014), therefore it is possible that inhibition of a single gene is insufficient to appreciably affect transport. Simultaneously knocking down Lrp1 and Megalin in all glia with Repo-Gal4 driver increased total sleep time (Figure 3A). To target Lrp1 and Megalin in barrier glia, and also to restrict the knockdown to the adult stage and thereby avoid developmental confounds, we used drivers specific to these glia and coupled them with the temperature-sensitive tubulin-Gal80 (tub-Gal80ts) system that suppresses Gal4 expression at 18 degrees but allows it at 31 degrees. When both Lrp genes were knocked down with barrier glia drivers, a significant increase in total sleep was seen with the PG driver (NP6923), but not with either of the SPG drivers (Moody, Rab9) (Figure 3B, C, D, E). Knockdown of Lrp1 and Megalin with the 9-137 driver, which expresses in both PG and SPG, also increased total sleep significantly (Figure 3C and D). Intriguingly, although the Repo driver did not yield a phenotype with Lrp1 alone, each of two Lrp1 RNAi constructs (GD 8397, GD 13913) expressed by the driver 9-137-Gal4 increased total sleep (Figure 3- figure supplement 1).

\section{Knockdown of Orct and Orct2 in barrier glia increases sleep}

The organic cation (Orct) transporters are multi-substrate transporters whose substrates include carnitine (Lahjouji, Mitchell et al. 2001), and perhaps also carnitylated molecules, based on in vitro evidence for Orct2 (Kou, Yao et al. 2017). As with the double knock down of Lrp1 and Megalin, knockdown of both Orct genes increased total sleep (Figure 4A). In fact, for the Orct genes, knockdown in either PG or SPG replicated the increased total sleep phenotype, although this was only true with one line for the SPG (Figure 4B). It 
is worth noting that the phenotypes with the barrier drivers are considerably more moderate than with knockdown in all glia.

A.

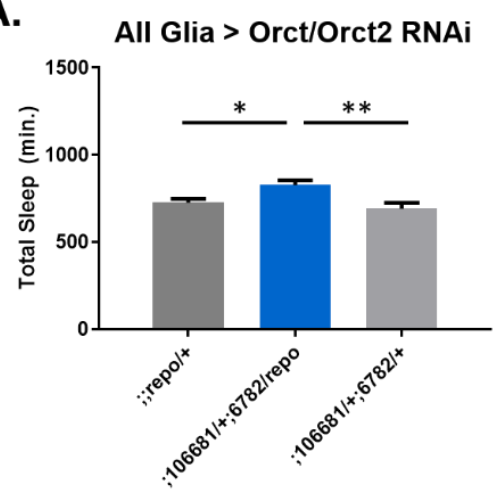

D.

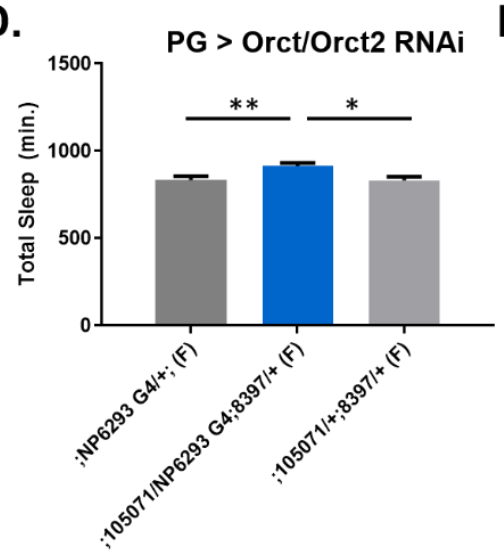

B.

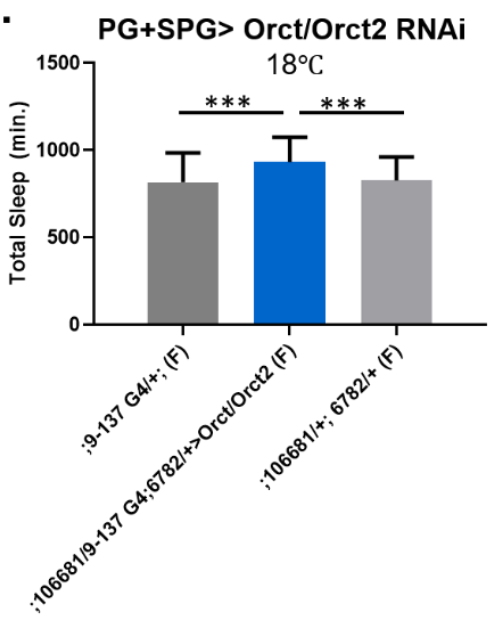

E.

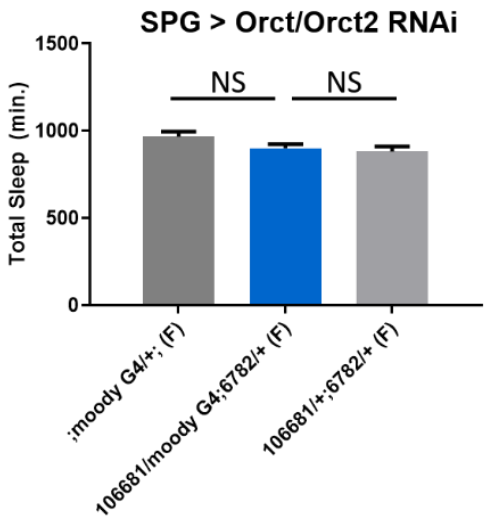

C.

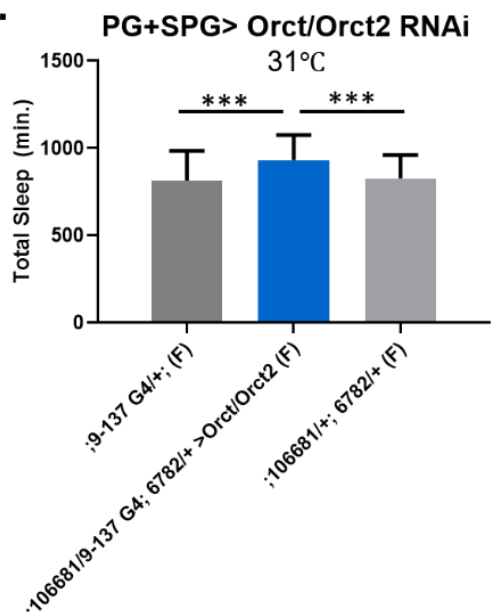

F.

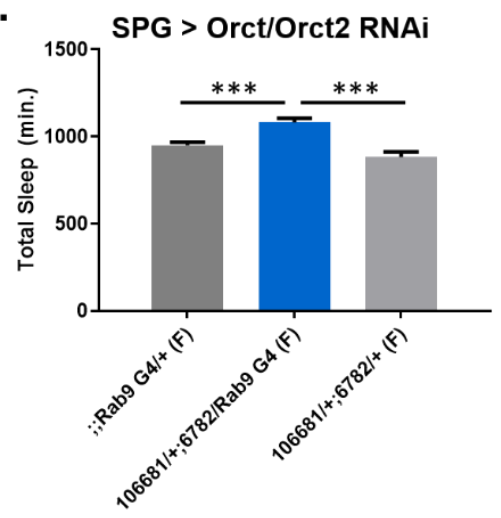

Figure 4. Sleep time changes with knockdown of Orct genes in all glia or barrier glia

RNAi constructs of Orct1 and Orct2 were expressed as follows: (A) Orct (6782 GD) and Orct2 (106681 KK) driven by Repo-GAL4; (B and C) Orct (6782 GD) and Orct2 (106681 KK) driven by (PG+SPG) 9-137-Gal4 at $18{ }^{\circ} \mathrm{C}$ (permissive), $n=30-32$ per genotype or at $31^{\circ} \mathrm{C}$ (restrictive), $n=30-32$ per genotype; (D-F) Orct (6782 GD) and Orct2 (106681 KK) driven by NP6293-GAL4, $n=13-16$ per genotype or by (SPG) moody-GAL4, $n=15-16$ per genotype or by (SPG) Rab9GAL4, $n=15-16$ per genotype. One-way ANOVA, with Holk-Sidak post-hoc comparisons. ${ }^{*} p<0.05,{ }^{* *} p<0.01,{ }^{* *} p<$ 0.001. Error bars represent standard error of the mean (SEM).

\section{Knockdown of Lrp and Orct genes in glia leads to accumulation of acylcarnitines}

Given that knockdown of the Lrp and Orct genes in glia parallels effects of Shi expression in terms of increasing total sleep, we asked if it had the same effect on metabolite accumulation in fly heads. Based 
on the metabolomic results of Repo>Shi, we chose to specifically assay acylcarnitines through LC-MS analysis. Metabolomic profiling requires considerable tissue, as so, as in the case of the Shit experiment, we collected heads from flies in which either Lrp1 and Megalin or Orct 1 and Orct2 were knocked down with Repo-Gal4.

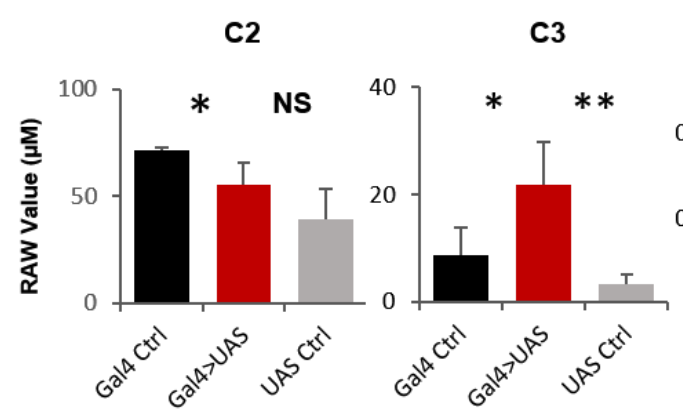

C14
C14:1
C4 Butyryl

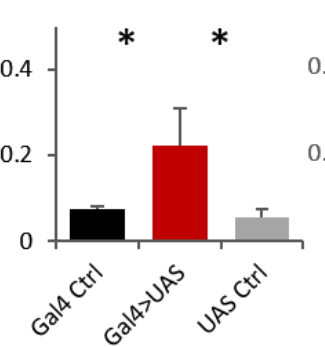

C16
C4-OH Isobutyryl

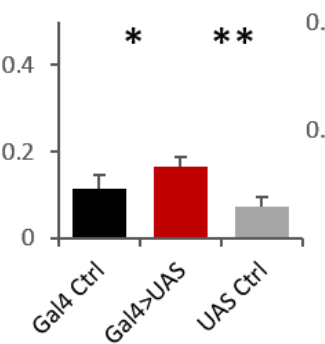

C16:1
C4 Isobutyryl

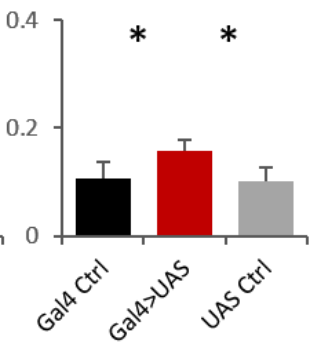

C18
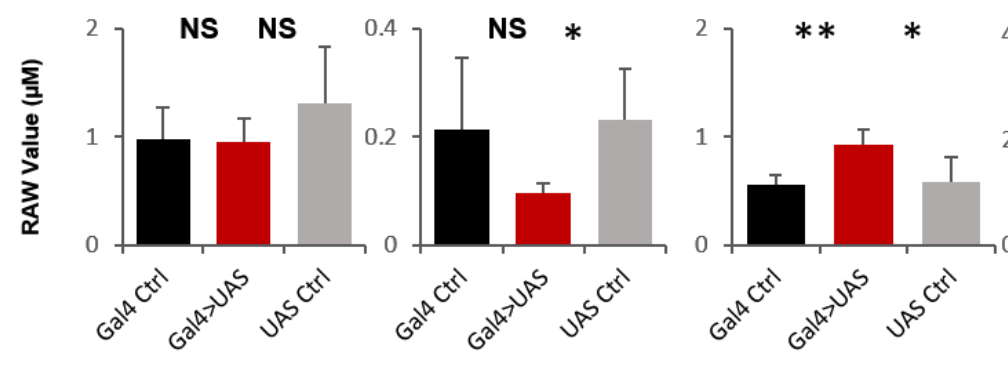

NS NS
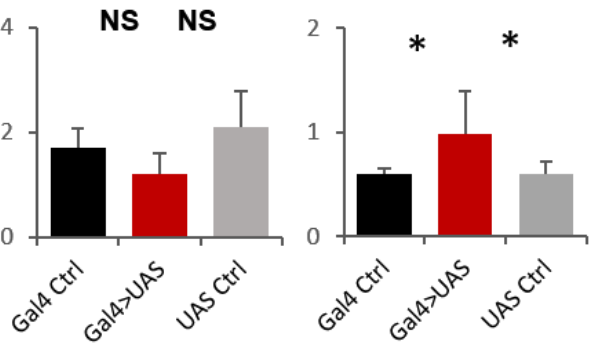

C18:1

C18:2

C20

C20:1

C22

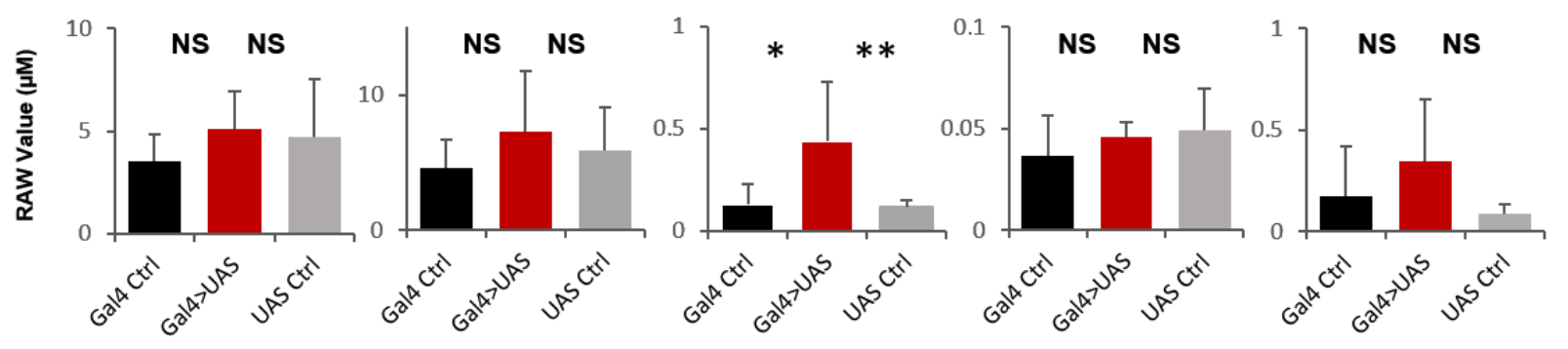

Figure 5. Acylcarnitine levels are increased in Repo>Lrp1+ Mgl fly heads

Short and medium chain length acylcarnitines from Repo Gal4 $>L r p 1+M g l$ fly heads and parental controls. The raw signal from LC/MS is plotted, $n=3$ samples, of 300 fly heads each. Student's t-test with comparison. ${ }^{*} p<0.05,{ }^{* *} p<$ 0.01. Error bars represent standard error of the mean (SEM).

Knockdown of Lrp1 and Megalin in all glia increased abundance in fly heads of the following acylcarnitine species: C3, C4 butyryl, C4-OH isobutyryl, C4 isobutyryl, C16, C18, C20 (Figure 5). Longer-chain acylcarnitines, e.g. those over $\mathrm{C22}$, were largely undetected in experimental samples. Knockdown of Orct and Orct2 similarly enriched acylcarnitines in fly heads. In particular, acylcarnitine species C16, C16:1 and 
C20 were increased significantly compared to their controls (Figure 6). Similar in the case of the Lrp samples, longer-chain acylcarnitines over 22 were not detected well.

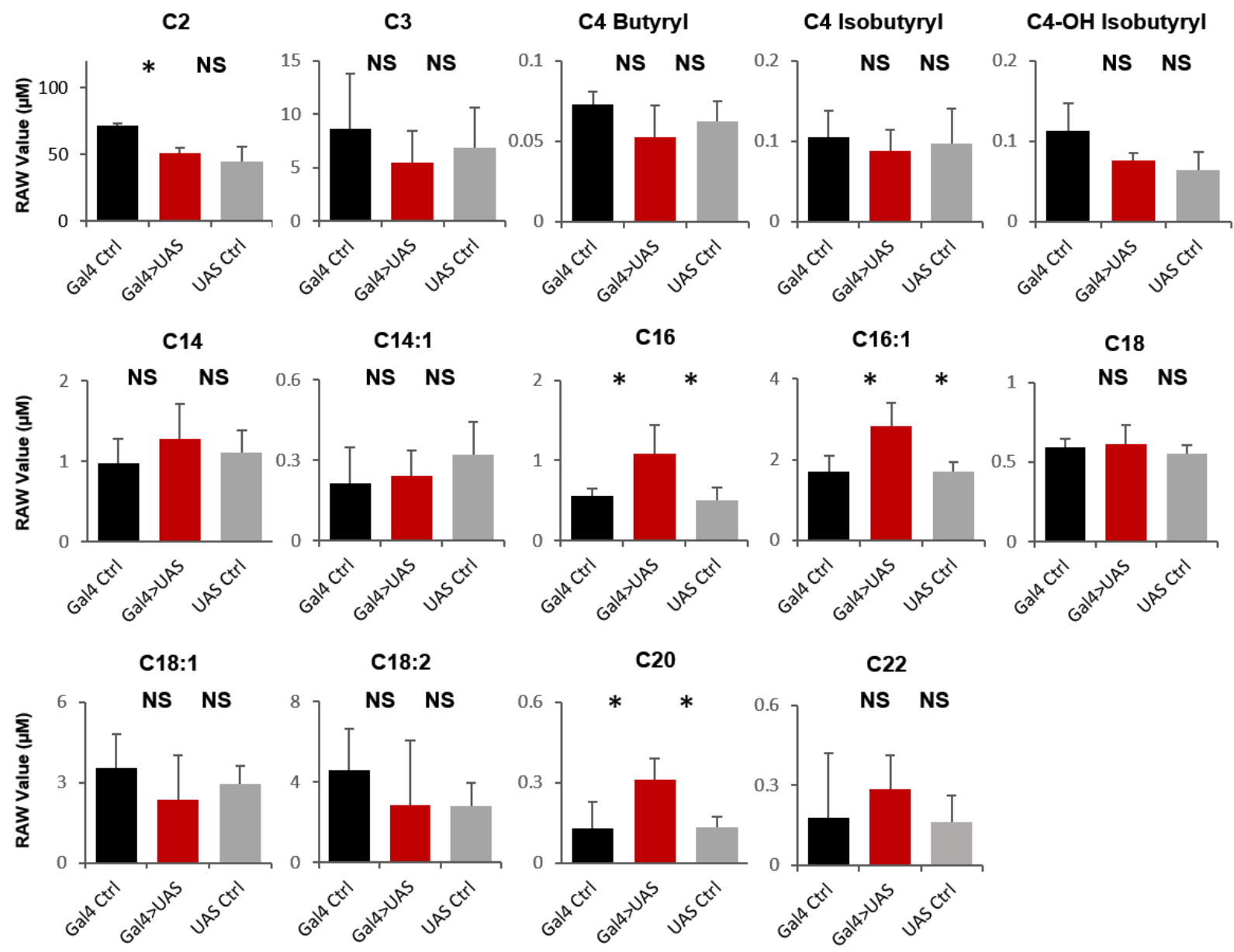

Figure 6. Acylcarnitine levels are increased in Repo> Orct+ Orct2 fly heads

Short and medium chain length acylcarnitines from Repo-Gal4 >Orct+Orct2 fly heads and parental controls. The raw signal from LC/MS is plotted, $n=3$ samples, of 300 fly heads each. Student's t-test with comparisons. ${ }^{*} p<0.05,{ }^{* *} p<$ 0.01. Error bars represent standard error of the mean (SEM). 


\section{Discussion:}

Our previous work suggested that endocytosis through the BBB is a function of sleep, but the nature of the molecules trafficked remained unknown. Using a dual-pronged approach of a targeted genetic screen and unbiased metabolomic analysis, we report here that the passage of lipids through the BBB is important for sleep. Blocking such transport increases sleep in conjunction with an accumulation of acylcarnitines.

Through a pan-glial RNAi knockdown screen of candidate genes expressed in the fly barrier, we identified molecules that affect daily sleep amount. In follow-up experiments, we targeted knockdown to each barrier layer separately, which is subject to the concern that behavioral phenotypes requiring simultaneous knockdown in both barrier populations would be missed. Nevertheless, we consider this scenario to be less probable, as permeability through the populations is quite different, with smaller solutes likely passing through the PG but not the tight barrier of the SPG, lipophilic solutes or xenobiotics potentially passing through each uninhibited, and larger solute requiring endocytic mechanisms that likely have to work in each population in tandem. Therefore, in most cases of knockdown, transport would either be inhibited by the one barrier population essential for it, or would be interrupted by either population. This was indeed the case for the lipid and carnitine transporters we focused on, where knockdown in a specific layer or either layer of the BBB was sufficient for a phenotype. While there was redundancy at the level of the transporters, the two barrier layers did not compensate for each other.

An additional consideration of preliminarily screening with pan-glial drivers is that if knockdown in multiple glial subtypes has opposing effects on sleep, we may have obscured a role for the barrier cells. Again, we attempted to minimize this risk by primarily selecting genes whose expression is both highly abundant and specifically enriched in the barrier populations, as opposed to the set of all glial cells in the transcriptome dataset (DeSalvo et al., 2014). The assumption is that multifold expression in the barrier populations is indicative of prevailing importance in these cells, although this is a caveat.

The vesicular monoamine transporter (VMAT) has previously been identified as a target of reserpine, which promotes sleep in the fly (Nall et al., 2014). VMAT mutants exhibit higher baseline sleep, and also lose less sleep than controls when subject to mechanical sleep deprivation. VMAT can traffic multiple monoamines such as dopamine, serotonin, histamine and octopamine, but no single neuronal population or neurotransmitter system was implicated as responsible for the VMAT sleep phenotype (Nall et al., 2014). 
In flies, VMAT exists as two isoforms, VMAT-A, which is expressed in monoaminergic neurons, and VMATB, which appears to be specific to perineurial glia (DeSalvo et al., 2014), as it is also found in fenestrated glia in the visual system (Romero-Calderon, Uhlenbrock et al. 2008), which are a specialized form of perineurial glia (Kremer, Jung et al. 2017). It is unknown whether VMAT-B would functional similarly in glia as VMAT-A does in neurons. VMAT-B contains an additional cytoplasmic domain, which has been suggested to promote retention in the plasma membrane as opposed to trafficking to vesicles (Greer, Grygoruk et al. 2005). VMAT knockdown increased sleep, as did disrupting endocytic trafficking at the barrier (Artiushin, Zhang et al. 2018). Whole-brain levels of monoamines were not altered in flies that expressed Shit in glia, nevertheless it is possible that this gross analysis would not be sensitive to local changes at the barrier. In the visual system glia, VMAT-B may be necessary for uptake of histamine (Romero-Calderon, Uhlenbrock et al. 2008). Interestingly, histamine is known to alter permeability of the blood-brain barrier in mammals (Lu, Diehl et al. 2010).

$\mathrm{Cln} 7$ is a major facilitator superfamily transporter implicated in neuronal ceroid lipofuscinoses, and hence considered to impact lysosomal/autophagal function (Siintola, Topcu et al. 2007). Neither the function, nor what this transporter traffics, are known, but it is thought to be vesicular as well, and is expressed in the perineurial glia in flies (Mohammed, O'Hare et al. 2017). Knockdown of Cln7 in all glia affected sleep in the opposite direction from knockdown only in the PG. One potential explanation would be that $\mathrm{Cln} 7$ acts on sleep in opposing ways in different glial populations, although protein expression data suggest that $C \ln 7$ is quite limited in the brain, and it is not clear whether it is in other glial populations.

Our metabolomic data indicated that acylcarnitines are elevated in the heads of the long-sleeping Repo>Shi ${ }^{1}$ flies. Acylcarnitines are transported to mitochondria for fatty acid oxidation, but are also secreted as they are found in plasma in mammals (Schooneman, Vaz et al. 2013). Given that circulating acylcarnitines can be taken up by cells, we investigated Lrp1/Megalin and Orct/Orct2 as candidate transporters for this uptake. Lrp1 and Megalin are lipoprotein carrier receptors known to function in the fly barrier (Brankatschk, Dunst et al. 2014) and although knockdown of each one separately in all glia did not affect sleep, reducing expression of both in all glia or barrier glia increased sleep. Likewise, knockdown of Orct and Orct2, which are homologs of the human carnitine transporters (Eraly and Nigam 2002) and transport carnitine as well as acylcarnitines (Pochini, Oppedisano et al. 2004), increases sleep. LC-MS 
analysis shows that knockdown of these transporters enriches acylcarnitines in fly heads just as blocking endocytosis does, supporting the idea that Lrp and Orct are among the proteins affected by the block in endocytosis. Exactly where the accumulation occurs is not known at this time, but we suggest that it is largely extracellular.

Acylcarnitine accumulation in flies with blocked glial endocytosis or lipid transport could occur as a consequence of the high sleep in these animals. We believe this is unlikely as an increase in acylcarnitines appears to generally occur under conditions of sleep deprivation i.e. conditions that would promote sleep. Thus, carnitine conjugation of long chain fatty acids was reported in cortical metabolites of sleepdeprived mice, while short and medium chain fatty acids were reduced (Hinard, Mikhail et al. 2012). Changes in acylcarnitines were also noted in the peripheral blood of sleep-deprived or sleep-restricted humans (Davies, Ang et al. 2014, Weljie, Meerlo et al. 2015) as well as over a day:night cycle (Dallman etal (Ang, Revell et al. 2012, Dallmann, Viola et al. 2012). We find too that a common feature of shortsleeping fly mutants, which are models for chronic sleep deprivation, is an increase in acylcarnitines, regardless of the mechanism that causes the sleep loss (Bedont, Kolesnik et al. 2021). Thus, acylcarnitines are generally associated with sleepiness. While this does not necessarily mean that they promote sleep, we suggest that acylcarnitines are a key marker of sleep need across species, and could be exploited for this purpose. 


\section{Methods}

\section{Fly Stocks}

The initial screen was performed with lab stock drivers: ;; Repo-GAL4/TM6c, Sb and UAS-Dicer; RepoGeneSwitch. SPG driver Moody-GAL4 and surface driver 9-137-Gal4 was shared by Roland Bainton, while PG driver NP6293-GAL4 was a gift of Marc Freeman. ;;UAS-20xShi.ts1 (referred to as UAS-20xShi') was shared by Gerald Rubin. Rab9-GAL4 (\#51587) was acquired from Bloomington. RNAi lines were ordered from VDRC (KK and GD collection) and Bloomington (TRiP collection) stock centers, with the stock number provided in Figure 1 and supplement Figure 1. For control genotypes, GAL4 and UAS lines were crossed to iso31.

\section{Behavior}

Flies were crossed and raised on standard food in bottles. Offspring were kept at $25{ }^{\circ} \mathrm{C}$, in LD12:12 conditions until at least 6 days post-eclosion, before age-matched flies which were group housed in bottles were used in sleep assays. Mated females were loaded into glass locomotor tubes with $2 \%$ agar $5 \%$ sugar. Sleep was quantified by the Drosophila Activity Monitor (DAM) system, by the established minimum definition of 5 minutes of inactivity. Data was analyzed in PySolo (Gilestro et al., 2009).

\section{Metabolomics}

Entire flies were quickly frozen in Falcon tubes chilled on dry ice, and placed at $-80^{\circ} \mathrm{C}$. Each tube contained 50 flies. Heads were subsequently removed from the body by briefly vortexing the tube. Heads were then separated from the rest of the body by an array of copper sieves, whose housing was buried in dry ice to keep the preparation cool. For each sample, 200 fly heads, of equal parts from males and females, were collected in $1.5 \mathrm{~mL}$ tubes which were quickly refrozen. Samples were shipped on dry ice to Metabolon, Inc., where they were assessed by LC-MS (Evans...Milgram 2009). For metabolomic analysis of Lrp and Orct knockdown fly lines, each sample contained 300 fly heads, of equal parts from males and females. Samples were processed by LC-MS at the Penn Metabolomics Core.

\section{Statistics:}

For both behavioral and acylcarnitine metabolomics results, the experimental group was compared to two parental controls by One-Way ANOVA with Holm-Sidak post-hoc tests. For the initial comparisons of metabolomics data, Metabolon performed Welch's t-tests on scaled signal data for each metabolite, 
between all conditions. Raw signal was scaled so that the median would be equal to 1, using all samples that had been concurrently run. Missing values were filled in with the lowest value of run samples for that metabolite. Additional details of statistics tests are listed in the figure legends.

\section{Acknowledgement}

We thank Dr. Chris Petucci and the Penn Metabolomics Core for providing measurements of acylcarnitines in fly heads.

Funding: The work was supported by HHMI and by R01DK120757 


\section{References}

Ang, J. E., V. Revell, A. Mann, S. Mäntele, D. T. Otway, J. D. Johnston, A. E. Thumser, D. J. Skene and F. Raynaud (2012). "Identification of human plasma metabolites exhibiting time-of-day variation using an untargeted liquid chromatography-mass spectrometry metabolomic approach." Chronobiology international 29(7): 868-881.

Artiushin, G., S. L. Zhang, H. Tricoire and A. Sehgal (2018). "Endocytosis at the Drosophila blood-brain barrier as a function for sleep." eLife 7: e43326.

Bedont, J., A. Kolesnik, D. Malik, A. Weljie and A. Sehgal (2021). "Polyamine elevation and nitrogen stress are toxic hallmarks of chronic sleep loss in <em>Drosophila melanogaster</em>." bioRxiv: 2021.2010.2001.462746.

Brankatschk, M., S. Dunst, L. Nemetschke and S. Eaton (2014). "Delivery of circulating lipoproteins to specific neurons in the Drosophila brain regulates systemic insulin signaling." Elife 3.

Cao, J., R. Albertson, B. Riggs, C. M. Field and W. Sullivan (2008). "Nuf, a Rab11 effector, maintains cytokinetic furrow integrity by promoting local actin polymerization." J Cell Biol 182(2): 301-313. Chell, J. M. and A. H. Brand (2010). "Nutrition-responsive glia control exit of neural stem cells from quiescence." Cell 143(7): 1161-1173.

Cheng, Z., M. Tsuda, Y. Kishita, Y. Sato and T. Aigaki (2013). "Impaired energy metabolism in a Drosophila model of mitochondrial aconitase deficiency." Biochem Biophys Res Commun 433(1): 145-150. Dallmann, R., A. U. Viola, L. Tarokh, C. Cajochen and S. A. Brown (2012). "The human circadian metabolome." Proceedings of the National Academy of Sciences of the United States of America 109(7): 2625-2629.

Davies, S. K., J. E. Ang, V. L. Revell, B. Holmes, A. Mann, F. P. Robertson, N. Cui, B. Middleton, K. Ackermann, M. Kayser, A. E. Thumser, F. I. Raynaud and D. J. Skene (2014). "Effect of sleep deprivation on the human metabolome." Proceedings of the National Academy of Sciences of the United States of America 111(29): 10761-10766.

DeSalvo, M. K., S. J. Hindle, Z. M. Rusan, S. Orng, M. Eddison, K. Halliwill and R. J. Bainton (2014). "The Drosophila surface glia transcriptome: evolutionary conserved blood-brain barrier processes." Frontiers in neuroscience 8: 346-346.

DeSalvo, M. K., S. J. Hindle, Z. M. Rusan, S. Orng, M. Eddison, K. Halliwill and R. J. Bainton (2014). "The Drosophila surface glia transcriptome: evolutionary conserved blood-brain barrier processes." $\underline{\text { Frontiers }}$ in neuroscience 8.

Eraly, S. A. and S. K. Nigam (2002). "Novel human cDNAs homologous to Drosophila Orct and mammalian carnitine transporters." Biochem Biophys Res Commun 297(5): 1159-1166.

Greer, C. L., A. Grygoruk, D. E. Patton, B. Ley, R. Romero-Calderon, H. Y. Chang, R. Houshyar, R. J. Bainton, A. Diantonio and D. E. Krantz (2005). "A splice variant of the Drosophila vesicular monoamine transporter contains a conserved trafficking domain and functions in the storage of dopamine, serotonin, and octopamine." J Neurobiol 64(3): 239-258.

Hediger, M. A., B. Clemencon, R. E. Burrier and E. A. Bruford (2013). "The ABCs of membrane transporters in health and disease (SLC series): introduction." Mol Aspects Med 34(2-3): 95-107. Herz, J. (2003). "LRP: a bright beacon at the blood-brain barrier." J Clin Invest 112(10): 1483-1485. Hinard, V., C. Mikhail, S. Pradervand, T. Curie, R. H. Houtkooper, J. Auwerx, P. Franken and M. Tafti (2012). "Key electrophysiological, molecular, and metabolic signatures of sleep and wakefulness revealed in primary cortical cultures." J Neurosci 32(36): 12506-12517.

Kou, L., Q. Yao, M. Sun, C. Wu, J. Wang, Q. Luo, G. Wang, Y. Du, Q. Fu, Z. He, V. Ganapathy and J. Sun (2017). "Cotransporting lon is a Trigger for Cellular Endocytosis of Transporter-Targeting Nanoparticles: A Case Study of High-Efficiency SLC22A5 (OCTN2)-Mediated Carnitine-Conjugated Nanoparticles for Oral Delivery of Therapeutic Drugs." Adv Healthc Mater 6(17). 
Kremer, M. C., C. Jung, S. Batelli, G. M. Rubin and U. Gaul (2017). "The glia of the adult Drosophila nervous system." Glia 65(4): 606-638.

Lahjouji, K., G. A. Mitchell and I. A. Qureshi (2001). "Carnitine transport by organic cation transporters and systemic carnitine deficiency." Mol Genet Metab 73(4): 287-297.

Lu, C., S. A. Diehl, R. Noubade, J. Ledoux, M. T. Nelson, K. Spach, J. F. Zachary, E. P. Blankenhorn and C. Teuscher (2010). "Endothelial histamine $\mathrm{H} 1$ receptor signaling reduces blood-brain barrier permeability and susceptibility to autoimmune encephalomyelitis." Proc Natl Acad Sci U S A 107(44): 18967-18972. Maniere, G., A. B. Ziegler, F. Geillon, D. E. Featherstone and Y. Grosjean (2016). "Direct Sensing of Nutrients via a LAT1-like Transporter in Drosophila Insulin-Producing Cells." Cell Rep 17(1): 137-148. Martin, J. F., E. Hersperger, A. Simcox and A. Shearn (2000). "minidiscs encodes a putative amino acid transporter subunit required non-autonomously for imaginal cell proliferation." Mech Dev 92(2): 155167.

Mohammed, A., M. B. O'Hare, A. Warley, G. Tear and R. I. Tuxworth (2017). "in vivo localization of the neuronal ceroid lipofuscinosis proteins, CLN3 and CLN7, at endogenous expression levels." Neurobiol Dis 103: 123-132.

Pochini, L., F. Oppedisano and C. Indiveri (2004). "Reconstitution into liposomes and functional characterization of the carnitine transporter from renal cell plasma membrane." Biochim Biophys Acta 1661(1): 78-86.

Romero-Calderon, R., G. Uhlenbrock, J. Borycz, A. F. Simon, A. Grygoruk, S. K. Yee, A. Shyer, L. C. Ackerson, N. T. Maidment, I. A. Meinertzhagen, B. T. Hovemann and D. E. Krantz (2008). "A glial variant of the vesicular monoamine transporter is required to store histamine in the Drosophila visual system." PLoS Genet 4(11): e1000245.

Schooneman, M. G., F. M. Vaz, S. M. Houten and M. R. Soeters (2013). "Acylcarnitines: reflecting or inflicting insulin resistance?" Diabetes 62(1): 1-8.

Siintola, E., M. Topcu, N. Aula, H. Lohi, B. A. Minassian, A. D. Paterson, X. Q. Liu, C. Wilson, U. Lahtinen, A. K. Anttonen and A. E. Lehesjoki (2007). "The novel neuronal ceroid lipofuscinosis gene MFSD8 encodes a putative lysosomal transporter." Am J Hum Genet 81(1): 136-146.

Speder, P. and A. H. Brand (2014). "Gap junction proteins in the blood-brain barrier control nutrientdependent reactivation of Drosophila neural stem cells." Dev Cell 30(3): 309-321.

Volkenhoff, A., A. Weiler, M. Letzel, M. Stehling, C. Klambt and S. Schirmeier (2015). "Glial Glycolysis Is Essential for Neuronal Survival in Drosophila." Cell Metab 22(3): 437-447.

Weiler, A., A. Volkenhoff, H. Hertenstein and S. Schirmeier (2017). "Metabolite transport across the mammalian and insect brain diffusion barriers." Neurobiol Dis 107: 15-31.

Weljie, A. M., P. Meerlo, N. Goel, A. Sengupta, M. S. Kayser, T. Abel, M. J. Birnbaum, D. F. Dinges and A. Sehgal (2015). "Oxalic acid and diacylglycerol 36:3 are cross-species markers of sleep debt." Proc Natl Acad Sci U S A 112(8): 2569-2574.

Wu, Y., X. Zheng, M. Zhang, A. He, Z. Li and X. Zhan (2010). "Cloning and functional expression of Rh50like glycoprotein, a putative ammonia channel, in Aedes albopictus mosquitoes." J Insect Physiol 56(11): 1599-1610. 
bioRxiv preprint doi: https://doi.org/10.1101/2022.02.17.480875; this version posted February 21, 2022. The copyright holder for this preprint

(which was not certified by peer review) is the author/funder, who has granted bioRxiv a license to display the preprint in perpetuity. It is made available under aCC-BY-NC-ND 4.0 International license.

\begin{tabular}{|c|c|c|c|c|c|c|c|c|c|}
\hline Sub Pathway & Biochemical Name & Platform & Comp ID & KEGG & HMDB & PubChem & $\frac{\text { Gal4>UAS }}{\text { GAL4 Ctrl }}$ & $\frac{\text { Gal4>UAS }}{\text { UAS CtrI }}$ & $\frac{\text { UAS CtrI }}{\text { GAL4 Ctrl }}$ \\
\hline \multirow{10}{*}{ Glycine, Serine and Threonine Metabolism } & glycine & LC/MS pos early & 58 & $\underline{\mathrm{C000037}}$ & HMDB00123 & 750 & 0.73 & 0.90 & 0.81 \\
\hline & \begin{tabular}{|l}
$\mathrm{N}$-acetylglycine \\
\end{tabular} & LCIMS polar & 27710 & & HMDB00532 & 10972 & 0.77 & 0.96 & 0.80 \\
\hline & sarcosine & LC/MS pos early & 1516 & $\underline{\mathrm{C00213}}$ & HMDB00271 & 1088 & 0.36 & 1.12 & 0.33 \\
\hline & betaine & LCIMS pos early & 3141 & $\underline{\underline{C 00719}}$ & HMDB00043 & 247 & 0.56 & 0.56 & 1.01 \\
\hline & serine & LC/MS pos early & 1648 & $\underline{\underline{000065}}$ & $\underline{\text { HMDB00187 }}$ & 5951 & 1.09 & 1.01 & 1.08 \\
\hline & $\mathrm{N}$-acetylserine & LC/MS pos early & 37076 & & HMDB02931 & 65249 & 0.87 & 1.02 & 0.85 \\
\hline & 2-methylserine & LC/MS pos early & 53229 & $\underline{\mathrm{C} 02115}$ & & 94309 & 0.75 & 0.50 & 1.49 \\
\hline & threonine & LC/MS pos early & 1284 & $\underline{\mathrm{CO0} 188}$ & HMDB00167 & 6288 & 0.72 & 0.94 & 0.77 \\
\hline & $\mathrm{N}$-acetytthreonine & LC/MS polar & 33939 & & & 152204 & 0.56 & 0.90 & 0.63 \\
\hline & homoserine & LC/MS pos early & 18351 & $\underline{\mathrm{C00263}}$ & HMDB00719 & 12647 & 1.27 & 0.79 & 1.61 \\
\hline \multirow{6}{*}{ Alanine and Aspartate Metabolism } & alanine & LC/MS pos early & 1126 & $\underline{\mathrm{CO00041}}$ & HMDB00161 & 5950 & 0.98 & 0.96 & 1.03 \\
\hline & \begin{tabular}{|l}
$\mathrm{N}$-acetylalanine \\
\end{tabular} & LCIMS polar & 1585 & $\underline{\underline{C 02847}}$ & HMDB00766 & 88064 & 0.73 & 0.89 & 0.82 \\
\hline & $\mathrm{N}$-methylalanine & LC/MS pos early & 37069 & $\underline{\mathrm{C02721}}$ & HMDB01906 & 5288725 & 0.59 & 0.63 & 0.93 \\
\hline & N-acetylaspartate (NAA) & LC/MS polar & 22185 & $\underline{\mathrm{C01042}}$ & HMDB00812 & 65065 & 1.46 & 1.12 & 1.30 \\
\hline & asparagine & LC/MS pos early & 512 & $\underline{\mathrm{C00152}}$ & HMDB00168 & 6267 & 1.11 & 0.81 & 1.37 \\
\hline & \begin{tabular}{|l|}
$\mathrm{N}$-acetylasparagine \\
\end{tabular} & LC/MS pos early & 33942 & & HMDB06028 & 99715 & 0.81 & 0.89 & 0.91 \\
\hline \multirow{11}{*}{ Glutamate Metabolism } & glutamate & LC/MS pos early & 57 & $\underline{\underline{C 00025}}$ & HMDB00148 & 611 & 1.41 & 1.06 & 1.33 \\
\hline & glutamine & LC/MS pos early & 53 & $\underline{c 00064}$ & HMDB00641 & 5961 & 1.32 & 0.87 & 1.51 \\
\hline & alpha-ketoglutaramate* & LCIMS polar & 62101 & & & & 1.84 & 0.96 & 1.92 \\
\hline & \begin{tabular}{|l}
$\mathrm{N}$-acetylglutamate \\
\end{tabular} & LCIMS polar & 15720 & $\underline{\underline{C 00624}}$ & HMDB01138 & 70914 & 1.27 & 0.61 & 2.08 \\
\hline & \begin{tabular}{|l}
$\mathrm{N}$-acetylglutamine \\
\end{tabular} & LC/MS pos early & 33943 & $\underline{\underline{C 02716}}$ & HMDB06029 & 182230 & 0.68 & 0.92 & 0.74 \\
\hline & glutamate, gamma-methyl ester & LC/MS pos early & 33487 & & HMDB61715 & 68662 & 0.80 & 0.86 & 0.93 \\
\hline & \begin{tabular}{|l} 
pyroglutamine \\
\end{tabular} & LC/MS pos early & 46225 & & & 134508 & 0.34 & 0.95 & 0.36 \\
\hline & gamma-aminobutyrate (GABA) & LC/MS pos early & 1416 & $\underline{\mathrm{C} 00334}$ & HMDB00112 & 119 & 1.14 & 1.03 & 1.11 \\
\hline & \begin{tabular}{|c|} 
carboxyethyl-GABA \\
\end{tabular} & LC/MS pos early & 40007 & & HMDB02201 & 2572 & 0.76 & 1.15 & 0.66 \\
\hline & N-methyl-GABA & LC/MS pos early & 39577 & $\underline{\mathrm{C} 15987}$ & & 70703 & 0.82 & 0.64 & 1.29 \\
\hline & propionylglutamine & LC/MS pos early & 54909 & & & & 0.65 & 1.05 & 0.62 \\
\hline \multirow{9}{*}{ Histidine Metabolism } & histidine & LCIMS neg & 59 & $\underline{\mathrm{C00135}}$ & HMDB00177 & 6274 & 0.84 & 0.81 & 1.04 \\
\hline & 1-methylhistidine & LC/MS pos early & 30460 & $\underline{\mathrm{C01152}}$ & HMDB00001 & 92105 & 1.11 & 0.73 & 1.51 \\
\hline & 3-methylhistidine & LC/MS pos early & 15677 & $\underline{\mathrm{C01152}}$ & HMDB00479 & 64969 & 1.91 & 0.26 & 7.23 \\
\hline & N-acety/histidine & LC/MS pos early & 33946 & $\underline{\mathrm{C02997}}$ & HMDB32055 & 75619 & 0.82 & 0.81 & 1.00 \\
\hline & imidazole propionate & LC/MS pos early & 40730 & & HMDB02271 & 70630 & 0.46 & 0.77 & 0.60 \\
\hline & imidazole lactate & LC/MS pos early & 15716 & $\underline{\underline{C 05568}}$ & HMDB02320 & 440129 & 0.57 & 0.75 & 0.75 \\
\hline & histamine & LC/MS pos early & 1574 & $\underline{\underline{C 00388}}$ & HMDB00870 & 774 & 1.05 & 0.93 & 1.13 \\
\hline & 4-imidazoleacetate & LC/MS pos early & 32349 & $\underline{\mathrm{C028335}}$ & HMDB02024 & 96215 & 0.86 & 0.92 & 0.94 \\
\hline & N-acetylhistamine & LC/MS pos early & 48679 & $\underline{\mathrm{C05135}}$ & HMDB13253 & 69602 & 1.92 & 1.64 & 1.17 \\
\hline \multirow{6}{*}{ Lysine Metabolism } & |ysine & LC/MS pos early & 1301 & $\underline{c 00047}$ & HMDB00182 & 5962 & 1.31 & 1.10 & 1.19 \\
\hline & NG,NG,N6-trimethyllysine & LC/MS pos early & 1498 & $\underline{\mathrm{CO} 3793}$ & HMDB01325 & 440120 & 0.96 & 1.96 & 0.49 \\
\hline & 5-(galactosylhydroxy)-L-lysine & LC/MS pos early & 43582 & & & & 1.05 & 0.88 & 1.19 \\
\hline & saccharopine & LCIMS polar & 1495 & C00449 & HMDB00279 & 160556 & 1.53 & 1.37 & 1.11 \\
\hline & pipecolate & LC/MS pos early & 1444 & $\underline{C 00408}$ & HMDB00070 & 849 & 0.74 & 1.22 & 0.61 \\
\hline & N-trimethyl 5-aminovalerate & LC/MS pos early & 57687 & & & & 3.87 & 1.87 & 2.07 \\
\hline
\end{tabular}


bioRxiv preprint doi: https://doi.org/10.1101/2022.02.17.480875; this version posted February 21, 2022. The copyright holder for this preprint

(which was not certified by peer review) is the author/funder, who has granted bioRxiv a license to display the preprint in perpetuity. It is made available under aCC-BY-NC-ND 4.0 International license.

\begin{tabular}{|c|c|c|c|c|c|c|c|c|c|}
\hline Sub Pathway & Biochemical Name & Platform & Comp ID & KEGG & HMDB & PubChem & $\frac{\text { Gal4>UAS }}{\text { GAL4 Ctrl }}$ & $\frac{\text { Gal } 4>\text { UAS }}{\text { UAS CtrI }}$ & $\frac{\text { UAS CtrI }}{\text { GAL4 CtrI }}$ \\
\hline \multirow{2}{*}{ Phenylalanine Metabolism } & phenylalanine & LC/MS pos early & 64 & $\underline{C 00079}$ & HMDB00159 & 6140 & 0.81 & 0.90 & 0.90 \\
\hline & \begin{tabular}{|l|}
$\mathrm{N}$-acetylphenylalanine \\
\end{tabular} & LC/MS neg & 33950 & $\underline{C 03519}$ & HMDB00512 & 74839 & 0.58 & 1.37 & 0.42 \\
\hline \multirow{3}{*}{ Tyrosine Metabolism } & tyrosine & LC/MS pos early & 1299 & $\underline{C 00082}$ & HMDB00158 & 6057 & 2.61 & 1.29 & 2.03 \\
\hline & dihydoxyphenylalanine (L-DOPA) & LC/MS pos early & 1576 & $\underline{\mathrm{CO00355}}$ & HMDB00181 & 6047 & 1.41 & 1.04 & 1.36 \\
\hline & $\mathrm{N}$-formylphenylalanine & LC/MS neg & 48433 & & & 759256 & 1.50 & 1.31 & 1.15 \\
\hline \multirow{6}{*}{ Tryptophan Metabolism } & tryptophan & LC/MS pos early & 54 & $\underline{C 00078}$ & HMDB00929 & 6305 & 1.17 & 1.02 & 1.15 \\
\hline & \begin{tabular}{|l|} 
kynurenine \\
\end{tabular} & LC/MS pos early & 15140 & C00328 & HMDB00684 & 161166 & 2.29 & 1.44 & 1.59 \\
\hline & kynurenate & LC/MS neg & 1417 & $\underline{C 01717}$ & HMDB00715 & 3845 & 3.21 & 2.14 & 1.50 \\
\hline & 3-hydroxykynurenine & LC/MS pos early & 22110 & $\underline{\mathrm{C} 02794}$ & HMDB00732 & 89 & 4.59 & 1.17 & 3.94 \\
\hline & xanthurenate & LC/MS neg & 15679 & $\underline{\mathrm{C} 02470}$ & HMDB00881 & 5699 & 2.05 & 1.07 & 1.91 \\
\hline & $\mathrm{N}$-acetylserotonin & LC/MS neg & 1500 & $\underline{\mathrm{C} 00978}$ & HMDB01238 & 903 & 0.81 & 0.99 & 0.82 \\
\hline \multirow{12}{*}{ Leucine, Isoleucine and Valine Metabolism } & leucine & LC/MS pos early & 60 & & & 5246661 & 0.64 & 0.95 & 0.68 \\
\hline & $\mathrm{N}$-acetylleucine & LC/MS neg & 1587 & $\mathrm{C} 02710$ & HMDB11756 & 70912 & 0.61 & 1.14 & 0.53 \\
\hline & 4-methyl-2-oxopentanoate & LC/MS neg & 22116 & $\mathrm{C} 00233$ & HMDB00695 & 70 & 0.61 & 1.17 & 0.52 \\
\hline & beta-hydroxyisovalerate & LC/MS polar & 12129 & & HMDB00754 & 69362 & 0.81 & 1.05 & 0.78 \\
\hline & isoleucine & LC/MS pos early & 1125 & $\underline{\mathrm{CO} 0407}$ & HMDB00172 & 6306 & 0.71 & 0.94 & 0.76 \\
\hline & 3-methyl-2-oxovalerate & LC/MS neg & 15676 & $\underline{C 00671}$ & HMDB03736 & 47 & 0.75 & 1.16 & 0.65 \\
\hline & alpha-hydroxyisovalerate & LC/MS polar & 46537 & & HMDB00407 & 99823 & 0.81 & 0.96 & 0.84 \\
\hline & ethylmalonate & LC/MS polar & 15765 & & HMDB00622 & 11756 & 0.81 & 0.83 & 0.98 \\
\hline & methylsuccinate & LC/MS polar & 15745 & & HMDB01844 & 10349 & 0.96 & 0.82 & 1.18 \\
\hline & valine & LC/MS neg & 1649 & $\underline{C 00183}$ & HMDB00883 & 6287 & 0.62 & 0.87 & 0.72 \\
\hline & 3-methyl-2-oxobutyrate & LC/MS polar & 44526 & $\underline{C 00141}$ & HMDB00019 & 49 & 0.88 & 1.28 & 0.68 \\
\hline & 3-hydroxyisobutyrate & LC/MS polar & 1549 & $\underline{\mathrm{C} 06001}$ & HMDB00336 & 87 & 0.72 & 1.09 & 0.66 \\
\hline \multirow{16}{*}{ Methionine, Cysteine, SAM and Taurine Metabolism } & methionine & LC/MS pos early & 1302 & $\underline{\mathrm{C} 00073}$ & HMDB00696 & 6137 & 0.75 & 1.08 & 0.70 \\
\hline & $\mathrm{N}$-acetylmethionine & LC/MS neg & 1589 & $\underline{\mathrm{C} 02712}$ & HMDB11745 & 448580 & 0.54 & 0.96 & 0.57 \\
\hline & $\mathrm{N}$-formylmethionine & LC/MS neg & 2829 & $\underline{\mathrm{C} 03145}$ & HMDB01015 & 439750 & 0.76 & 1.53 & 0.50 \\
\hline & methionine sulfoxide & LC/MS pos early & 18374 & $\underline{\mathrm{C} 02989}$ & HMDB02005 & 158980 & 0.57 & 0.75 & 0.77 \\
\hline & $\mathrm{N}$-acetylmethionine sulfoxide & LC/MS pos early & 45428 & & & 193368 & 0.60 & 0.90 & 0.67 \\
\hline & S-adenosylhomocysteine (SAH) & LC/MS neg & 42382 & $\underline{C 00021}$ & HMDB00939 & 439155 & 1.14 & 1.22 & 0.93 \\
\hline & cystathionine & LC/MS pos early & 15705 & $\underline{\mathrm{C} 02291}$ & HMDB00099 & 439258 & 0.97 & 1.14 & 0.85 \\
\hline & cysteine & LC/MS pos early & 1868 & $\underline{\mathrm{C} 00097}$ & HMDB00574 & 5862 & 0.49 & 0.73 & 0.67 \\
\hline & $\mathrm{N}$-acetylcysteine & LC/MS pos early & 1586 & $\underline{\mathrm{C} 06809}$ & HMDB01890 & 12035 & 0.87 & 0.89 & 0.97 \\
\hline & S-methylcysteine sulfoxide & LC/MS pos early & 43378 & & HMDB29432 & 82142 & 1.12 & 0.71 & 1.58 \\
\hline & cystine & LC/MS neg & 56 & $\underline{\mathrm{C} 00491}$ & HMDB00192 & 67678 & 0.63 & 0.64 & 1.00 \\
\hline & lanthionine & LC/MS pos early & 42002 & & & 98504 & 0.75 & 1.48 & 0.50 \\
\hline & cysteine sulfinic acid & LC/MS pos early & 37443 & $\underline{\mathrm{C} 00606}$ & HMDB00996 & 109 & 1.84 & 0.87 & 2.12 \\
\hline & taurine & LC/MS neg & 2125 & $\underline{C 00245}$ & HMDB00251 & 1123 & 0.96 & 0.88 & 1.09 \\
\hline & $\mathrm{N}$-acetyltaurine & LC/MS neg & 48187 & & & 159864 & 1.02 & 0.85 & 1.20 \\
\hline & cyano-alanine & LC/MS polar & 35660 & $\underline{\mathrm{C} 02512}$ & & 13538 & 1.00 & 0.77 & 1.31 \\
\hline \multirow{12}{*}{ Urea cycle; Arginine and Proline Metabolism } & arginine & LC/MS pos early & 1638 & $\underline{\mathrm{C} 00062}$ & HMDB00517 & 232 & 0.99 & 0.96 & 1.03 \\
\hline & argininosuccinate & LC/MS pos early & 15497 & $\mathrm{CO} 03406$ & HMDB00052 & 828 & 1.11 & 1.23 & 0.90 \\
\hline & ornithine & LC/MS pos early & 1493 & $\underline{\mathrm{C} 00077}$ & HMDB03374 & 6262 & 1.38 & 1.38 & 1.00 \\
\hline & 2-oxoarginine* ${ }^{*}$ & LC/MS pos early & 55072 & $\mathrm{C} 03771$ & HMDB04225 & 558 & 0.73 & 1.00 & 0.74 \\
\hline & citrulline & LC/MS pos early & 2132 & $\underline{C 00327}$ & HMDB00904 & 9750 & 0.73 & 0.97 & 0.75 \\
\hline & proline & LC/MS pos early & 1898 & $\underline{C 00148}$ & HMDB00162 & 145742 & 1.31 & 1.02 & 1.28 \\
\hline & dimethylarginine (SDMA + ADMA) & LC/MS pos early & 36808 & $\underline{\mathrm{C} 03626}$ & HMDB01539 & 123831 & 1.38 & 1.09 & 1.27 \\
\hline & $\mathrm{N}$-acetylarginine & LC/MS pos early & 33953 & $\underline{\mathrm{C} 02562}$ & HMDB04620 & 67427 & 1.72 & 1.72 & 1.00 \\
\hline & $\mathrm{N}$-delta-acetylornithine & LC/MS pos early & 43249 & & & 9920500 & 1.35 & 1.09 & 1.24 \\
\hline & $\mathrm{N}$-alpha-acetylornithine & LC/MS pos early & 32984 & $\underline{\mathrm{C} 00437}$ & HMDB03357 & 439232 & 1.54 & 1.48 & 1.04 \\
\hline & trans-4-hydroxyproline & LC/MS pos early & 32306 & $\mathrm{C} 01157$ & HMDB00725 & 5810 & 0.77 & 0.62 & 1.23 \\
\hline & argininate $^{*}$ & LC/MS pos early & 57461 & & HMDB03148 & 160437 & 0.75 & 1.15 & 0.65 \\
\hline
\end{tabular}


bioRxiv preprint doi: https://doi.org/10.1101/2022.02.17.480875; this version posted February 21, 2022. The copyright holder for this preprint

(which was not certified by peer review) is the author/funder, who has granted bioRxiv a license to display the preprint in perpetuity. It is made available under aCC-BY-NC-ND 4.0 International license.

\begin{tabular}{|c|c|c|c|c|c|c|c|c|c|}
\hline Sub Pathway & Biochem ical Name & Platform & Comp ID & KEGG & HMDB & PubChem & $\frac{\text { Gal4>UAS }}{\text { GAL4 Ctrl }}$ & $\frac{\text { Gal } 4>\text { UAS }}{\text { UAS CtrI }}$ & $\frac{\text { UAS Ctrl }}{\text { GAL4 CtrI }}$ \\
\hline \multirow{6}{*}{ Polyamine Metabolism } & putrescine & LC/MS pos early & 1408 & $\underline{C 00134}$ & HMDB01414 & 1045 & 5.09 & 0.83 & 6.14 \\
\hline & $\mathrm{N}$-acetyl-isoputreanine ${ }^{*}$ & LC/MS pos early & 62309 & & & & 1.00 & 1.00 & 1.00 \\
\hline & spermidine & LC/MS pos early & 485 & $\underline{\mathrm{C} 00315}$ & HMDB01257 & 1102 & 1.23 & 0.99 & 1.25 \\
\hline & 5-methylthioadenosine (MTA) & LC/MS pos early & 1419 & $\underline{C 00170}$ & HMDB01173 & 439176 & 1.48 & 1.17 & 1.26 \\
\hline & 4-acetamidobutanoate & LC/MS pos early & 1558 & $\underline{\mathrm{C} 02946}$ & HMDB03681 & 18189 & 0.89 & 0.86 & 1.03 \\
\hline & $(\mathrm{N}(1)+\mathrm{N}(8))$-acetylspermidine & LC/MS pos early & 57814 & & & & 0.79 & 0.91 & 0.86 \\
\hline Guanidino and Acetamido Metabolism & 4-guanidinobutanoate & LC/MS pos early & 15681 & $\underline{\mathrm{C} 01035}$ & HMDB03464 & 500 & 1.00 & 1.14 & 0.87 \\
\hline \multirow{5}{*}{ Glutathione Metabolism } & glutathione, reduced (GSH) & LC/MS pos early & 2127 & $\underline{C 00051}$ & HMDB00125 & 124886 & 4.09 & 0.83 & 4.94 \\
\hline & cysteine-glutathione disulfide & LC/MS pos early & 35159 & & HMDB00656 & 4247235 & 1.72 & 0.82 & 2.10 \\
\hline & cysteinylglycine & LC/MS pos early & 35637 & $\underline{\mathrm{C} 01419}$ & $\underline{\mathrm{HMDB} 00078}$ & 439498 & 0.36 & 0.49 & 0.73 \\
\hline & cysteinylglycine disulfide* & LC/MS pos early & 62103 & & HMDB00709 & & 1.89 & 0.88 & 2.15 \\
\hline & 5-oxoproline & LC/MS neg & 1494 & C01879 & HMDB00267 & 7405 & 0.64 & 0.79 & 0.80 \\
\hline \multirow{12}{*}{ Gamma-glutamyl Amino Acid } & gamma-glutamylalanine & LC/MS pos early & 37063 & & HMDB29142 & 440103 & 0.88 & 0.91 & 0.97 \\
\hline & gamma-glutamylcysteine & LC/MS pos early & 1778 & $\mathrm{C} 00669$ & HMDB01049 & 842 & 0.25 & 0.58 & 0.43 \\
\hline & gamma-glutamylglutamate & LC/MS pos early & 36738 & $\underline{\mathrm{C} 05282}$ & HMDB11737 & 92865 & 0.90 & 0.83 & 1.08 \\
\hline & gamma-glutamylglutamine & LC/MS pos early & 2730 & $\underline{\mathrm{C} 05283}$ & HMDB11738 & 150914 & 1.66 & 1.13 & 1.47 \\
\hline & gamma-glutamylglycine & LC/MS pos early & 33949 & & HMDB11667 & 165527 & 0.37 & 0.63 & 0.59 \\
\hline & gamma-glutamylhistidine & LC/MS pos early & 18245 & & & 7017195 & 0.55 & 0.75 & 0.73 \\
\hline & gamma-glutamylisoleucine* & LC/MS neg & 34457 & & HMDB11170 & 14253342 & 0.40 & 1.01 & 0.39 \\
\hline & gamma-glutamylleucine & LC/MS neg & 18369 & & HMDB11171 & 151023 & 0.45 & 0.87 & 0.52 \\
\hline & gamma-glutamyl-alpha-lysine & LC/MS pos early & 55015 & & & 65254 & 1.29 & 1.32 & 0.98 \\
\hline & gamma-glutamylmethionine & LC/MS pos early & 44872 & & HMDB29155 & 7009567 & 0.43 & 1.40 & 0.31 \\
\hline & gamma-glutamylthreonine & LC/MS pos early & 33364 & & HMDB29159 & 76078708 & 0.61 & 0.94 & 0.65 \\
\hline & gamma-glutamylvaline & LC/MS pos early & 43829 & & HMDB11172 & 7015683 & 0.48 & 0.70 & 0.69 \\
\hline \multirow{43}{*}{ Dipeptide } & alanylalanine & LC/MS pos early & 15129 & & HMDB28680 & 5484352 & 1.13 & 0.91 & 1.24 \\
\hline & alanylglutamate & LC/MS pos early & 37064 & & & 656476 & 0.84 & 0.84 & 1.00 \\
\hline & alany/proline & LC/MS pos early & 37083 & & HMDB28695 & 418040 & 0.83 & 1.21 & 0.69 \\
\hline & alanylthreonine & LC/MS pos early & 37085 & & HMDB28697 & 426318 & 1.05 & 0.98 & 1.06 \\
\hline & alpha-glutamylalanine & LC/MS pos early & 41369 & & HMDB03764 & 100098 & 0.90 & 0.93 & 0.97 \\
\hline & alpha-glutamy Iglutamate & LC/MS pos early & 22166 & $\underline{\mathrm{C} 01425}$ & HMDB28818 & 439500 & 0.87 & 1.07 & 0.81 \\
\hline & asparaginylalanine & LC/MS pos early & 54731 & & HMDB28724 & & 1.12 & 0.98 & 1.14 \\
\hline & glutaminylglutamate & LC/MS pos early & 43025 & & & & 0.71 & 0.94 & 0.76 \\
\hline & serylthreonine & LC/MS pos early & 54732 & & HMDB29049 & & 0.69 & 0.92 & 0.74 \\
\hline & \begin{tabular}{|l} 
glycylglycine \\
\end{tabular} & LC/MS pos early & 21029 & $\underline{\mathrm{C} 02037}$ & HMDB11733 & 11163 & 0.86 & 1.05 & 0.82 \\
\hline & glycylisoleucine & LC/MS pos early & 36659 & & HMDB28844 & 88079 & 1.04 & 0.88 & 1.18 \\
\hline & glycylleucine & LC/MS pos early & 34398 & $\mathrm{C} 02155$ & HMDB00759 & 92843 & 0.89 & 0.83 & 1.07 \\
\hline & glycylphenylalanine & LC/MS neg & 33954 & & HMDB28848 & 92953 & 1.24 & 1.19 & 1.04 \\
\hline & glycylproline & LC/MS pos early & 22171 & & HMDB00721 & 3013625 & 0.69 & 0.75 & 0.92 \\
\hline & glycylvaline & LC/MS pos early & 18357 & & HMDB28854 & 97417 & 0.67 & 0.83 & 0.81 \\
\hline & isoleucylalanine & LC/MS pos early & 40046 & & HMDB28900 & 5246009 & 1.72 & 0.99 & 1.73 \\
\hline & isoleucylglutamate & LC/MS pos early & 40057 & & & & 0.96 & 1.23 & 0.79 \\
\hline & isoleucylglutamine & LC/MS pos early & 40019 & & & 7020102 & 1.60 & 1.05 & 1.52 \\
\hline & isoleucylglycine & LC/MS neg & 40008 & & HMDB28907 & 342532 & 1.15 & 1.09 & 1.05 \\
\hline & isoleucylthreonine & LC/MS pos early & 42968 & & HMDB28917 & 16122515 & 1.46 & 0.95 & 1.53 \\
\hline & leucylalanine & LC/MS pos early & 40010 & & HMDB28922 & 259321 & 4.22 & 1.01 & 4.19 \\
\hline & leucylglycine & LC/MS pos early & 40045 & & HMDB28929 & 79070 & 1.67 & 0.96 & 1.73 \\
\hline & leucylleucine & LC/MS pos early & 36756 & C11332 & HMDB28933 & 76807 & 5.53 & 0.92 & 6.02 \\
\hline & leucylproline & LC/MS pos early & 35663 & & HMDB11175 & 80817 & 0.52 & 0.94 & 0.56 \\
\hline & \begin{tabular}{|l|} 
leucylthreonine \\
\end{tabular} & LC/MS pos early & 42969 & & HMDB28939 & 10353878 & 2.16 & 0.91 & 2.37 \\
\hline & \begin{tabular}{|l|} 
phenylalanylglutamate \\
\end{tabular} & LC/MS neg & 41432 & & & 4422358 & 0.87 & 0.91 & 0.96 \\
\hline & \begin{tabular}{|l|} 
prolylalanine \\
\end{tabular} & LC/MS pos early & 40705 & & HMDB29010 & 418041 & 0.87 & 0.96 & 0.90 \\
\hline & prolylglutamine & LC/MS pos early & 40659 & & & & 1.11 & 0.92 & 1.21 \\
\hline & prolylglycine & LC/MS pos early & 40703 & & HMDB11178 & 6426709 & 0.86 & 1.01 & 0.85 \\
\hline & \begin{tabular}{|l|} 
prolylleucine \\
\end{tabular} & LC/MS pos early & 31914 & & & 3527720 & 0.94 & 0.92 & 1.03 \\
\hline & \begin{tabular}{|l|} 
proly|proline \\
\end{tabular} & LC/MS pos early & 40731 & & HMDB11180 & 11622593 & 0.71 & 0.89 & 0.80 \\
\hline & prolylthreonine & LC/MS pos early & 44551 & & HMDB29027 & & 0.74 & 1.12 & 0.67 \\
\hline & \begin{tabular}{|l|} 
prolylvaline \\
\end{tabular} & LC/MS pos early & 40720 & & & 152307 & 0.72 & 0.81 & 0.89 \\
\hline & serylalanine & LC/MS pos early & 42049 & & HMDB29032 & 17958834 & 1.07 & 0.85 & 1.26 \\
\hline & serylleucine & LC/MS pos early & 40066 & & HMDB29043 & 7015695 & 1.15 & 1.11 & 1.04 \\
\hline & serylproline & LC/MS pos early & 42055 & & HMDB29047 & 4369021 & 1.04 & 0.90 & 1.16 \\
\hline & serylserine & LC/MS pos early & 42053 & & & 138784 & 0.94 & 0.83 & 1.13 \\
\hline & serylvaline & LC/MS pos early & 42058 & & HMDB29052 & 7020159 & 1.45 & 0.95 & 1.54 \\
\hline & valylaspartate & LC/MS pos early & 40650 & & HMDB29123 & 9964657 & 0.91 & 0.92 & 0.99 \\
\hline & valylglycine & LC/MS neg & 40475 & & HMDB29127 & 136487 & 1.07 & 1.01 & 1.06 \\
\hline & valy|proline & LC/MS pos early & 40485 & & HMDB29135 & 5003412 & 0.70 & 0.95 & 0.74 \\
\hline & isoleucylleucine/leucylisoleucine & LC/MS pos early & 52322 & & & & 2.09 & 0.79 & 2.65 \\
\hline & alpha-glutamy|proline* & LC/MS pos early & 57731 & & & & 1.00 & 1.00 & 1.00 \\
\hline Modified Peptides & pyroglutamylleucine* ${ }^{*}$ & LC/MS neg & 62096 & & & & 0.94 & 1.26 & 0.75 \\
\hline
\end{tabular}


bioRxiv preprint doi: https://doi.org/10.1101/2022.02.17.480875; this version posted February 21, 2022. The copyright holder for this preprint (which was not certified by peer review) is the author/funder, who has granted bioRxiv a license to display the preprint in perpetuity. It is made available under aCC-BY-NC-ND 4.0 International license.

\begin{tabular}{|c|c|c|c|c|c|c|c|c|c|}
\hline Sub Pathway & Biochemical Name & Platform & Comp ID & KEGG & HMDB & PubChem & $\frac{\text { Gal4>UAS }}{\text { GAL4 Ctrl }}$ & $\frac{\text { Gal4 }>\text { UAS }}{\text { UAS CtrI }}$ & $\underline{\text { UAS Ctrl }}$ \\
\hline \multirow{8}{*}{ Glycolysis, Gluconeogenesis, and Pyruvate Metaboli } & glucose & LC/MS polar & 48152 & $\underline{\mathrm{C} 00031}$ & HMDB00122 & 79025 & 1.03 & 1.01 & 1.01 \\
\hline & fructose 1,6-diphosphate/glucose 1,6-diphosphate/m & LC/MS neg & 46896 & $\underline{\mathrm{C} 00354}$ & & & 1.62 & 0.84 & 1.93 \\
\hline & dihydroxyacetone phosphate (DHAP) & LC/MS neg & 15522 & $\underline{\mathrm{C} 00111}$ & HMDB01473 & 668 & 1.48 & 1.68 & 0.88 \\
\hline & 3-phosphoglycerate & LC/MS neg & 1414 & $\underline{\mathrm{C} 00597}$ & HMDB00807 & 724 & 0.86 & 0.81 & 1.06 \\
\hline & phosphoenolpyruvate (PEP) & LC/MS neg & 597 & $\underline{\mathrm{C} 00074}$ & HMDB00263 & 1005 & 0.77 & 0.73 & 1.06 \\
\hline & pyruvate & LC/MS polar & 48990 & $\underline{\mathrm{C} 00022}$ & HMDB00243 & 1060 & 1.01 & 0.86 & 1.17 \\
\hline & lactate & LC/MS polar & 527 & $\underline{\mathrm{C} 00186}$ & HMDB00190 & 612 & 0.63 & 0.85 & 0.74 \\
\hline & glycerate & LC/MS polar & 1572 & $\underline{\mathrm{C} 00258}$ & HMDB00139 & 752 & 1.04 & 1.12 & 0.93 \\
\hline \multirow{2}{*}{ Pentose Phosphate Pathw ay } & 6-phosphogluconate & LC/MS neg & 15442 & $\underline{\mathrm{C} 00345}$ & HMDB01316 & 91493 & 0.60 & 0.71 & 0.86 \\
\hline & sedoheptulose-7-phosphate & LC/MS neg & 35649 & $\underline{\mathrm{C} 05382}$ & HMDB01068 & 616 & 0.87 & 0.77 & 1.13 \\
\hline \multirow{8}{*}{ Pentose Metabolism } & ribose & LC/MS polar & 1471 & $\underline{\mathrm{C} 00121}$ & HMDB00283 & 5779 & 0.94 & 1.01 & 0.94 \\
\hline & ribitol & LC/MS polar & 15772 & $\underline{\mathrm{C} 00474}$ & HMDB00508 & 6912 & 0.01 & 0.87 & 0.02 \\
\hline & ribonate & LC/MS polar & 27731 & $\underline{\mathrm{C} 01685}$ & HMDB00867 & 5460677 & 0.78 & 0.91 & 0.86 \\
\hline & arabitol/xylitol & LC/MS polar & 48885 & $\underline{\mathrm{C} 01904}$ & & 6912 & 1.10 & 0.96 & 1.15 \\
\hline & ribulose/xylulose & LC/MS polar & 54671 & & & 5289590 & 0.74 & 0.94 & 0.79 \\
\hline & arabonate/xylonate & LC/MS polar & 48255 & & & & 0.93 & 0.93 & 1.00 \\
\hline & sedoheptulose & LC/MS polar & 53237 & & HMDB03219 & 5459879 & 0.84 & 0.75 & 1.13 \\
\hline & \begin{tabular}{|l} 
ribulonate/xylulonate \\
\end{tabular} & LC/MS polar & 61858 & & & & 1.01 & 1.00 & 1.01 \\
\hline \multirow{4}{*}{ Glycogen Metabolism } & maltotetraose & LC/MS polar & 15910 & $\underline{\mathrm{CO} 2052}$ & HMDB01296 & 446495 & 0.68 & 0.74 & 0.91 \\
\hline & maltotriose & LC/MS polar & 44688 & $\underline{\mathrm{C} 01835}$ & HMDB01262 & 439586 & 1.04 & 0.83 & 1.25 \\
\hline & maltose & LC/MS polar & 15586 & $\underline{\mathrm{C} 00208}$ & HMDB00163 & 10991489 & 1.26 & 0.90 & 1.40 \\
\hline & isomaltose & LC/MS polar & 39777 & $\underline{\mathrm{C} 00252}$ & HMDB02923 & 439193 & 1.02 & 1.13 & 0.91 \\
\hline \multirow{5}{*}{ Fructose, Mannose and Galactose Metabolism } & fructose & LC/MS polar & 48195 & $\underline{\mathrm{CO00095}}$ & HMDB00660 & 5984 & 0.85 & 0.90 & 0.94 \\
\hline & mannitol/sorbitol & LC/MS polar & 46142 & $\underline{\mathrm{C} 00794}$ & HMDB00247 & 5780 & 0.66 & 0.93 & 0.71 \\
\hline & mannose & LC/MS polar & 48153 & $\underline{\mathrm{C} 00159}$ & HMDB00169 & 18950 & 0.71 & 0.62 & 1.15 \\
\hline & galactitol (dulcitol) & LC/MS polar & 1117 & $\underline{\mathrm{C} 01697}$ & HMDB00107 & 11850 & 0.81 & 0.64 & 1.27 \\
\hline & galactonate & LC/MS polar & 27719 & $\underline{\mathrm{C} 00880}$ & HMDB00565 & 128869 & 0.66 & 0.64 & 1.03 \\
\hline Nucleotide Sugar & UDP-N-acetylglucosamine/galactosamine & LC/MS neg & 46148 & & & & 0.81 & 0.81 & 1.00 \\
\hline \multirow{5}{*}{ Aminosugar Metabolism } & glucuronate & LC/MS polar & 15443 & $\underline{\mathrm{C} 00191}$ & HMDB00127 & 444791 & 0.75 & 0.95 & 0.79 \\
\hline & $\mathrm{N}$-acetylglucosamine 6-phosphate & LC/MS polar & 15107 & $\underline{\mathrm{C} 00357}$ & HMDB02817 & 439219 & 2.39 & 0.90 & 2.67 \\
\hline & $\mathrm{N}$-acetylglucosaminylasparagine & LC/MS pos early & 48149 & $\underline{\mathrm{C} 04540}$ & HMDB00489 & 123826 & 1.26 & 0.93 & 1.36 \\
\hline & erythronate $^{*}$ & LC/MS polar & 42420 & & HMDB00613 & 2781043 & 0.90 & 0.98 & 0.91 \\
\hline & $\mathrm{N}$-acetylglucosamine/N-acetylgalactosamine & LC/MS pos early & 46539 & & HMDB00215 & 24139 & 0.87 & 0.93 & 0.94 \\
\hline \begin{tabular}{|l|} 
Advanced Glycation End-product \\
\end{tabular} & N6-carboxymethyllysine & LC/MS pos early & 36713 & & & 123800 & 1.06 & 0.97 & 1.09 \\
\hline \multirow{11}{*}{ TCA Cycle } & citrate & LC/MS neg & 1564 & $\underline{\mathrm{C} 00158}$ & HMDB00094 & 311 & 0.85 & 1.18 & 0.72 \\
\hline & aconitate [cis or trans] & LC/MS neg & 46173 & & & & 0.95 & 0.92 & 1.03 \\
\hline & isocitric lactone & LC/MS polar & 54724 & & & 98259 & 1.59 & 1.85 & 0.86 \\
\hline & alpha-ketoglutarate & LC/MS polar & 528 & $\underline{\mathrm{C} 00026}$ & HMDB00208 & 51 & 1.35 & 1.07 & 1.26 \\
\hline & succinate & LC/MS polar & 1437 & $\underline{\mathrm{C} 00042}$ & HMDB00254 & 1110 & 1.24 & 0.87 & 1.41 \\
\hline & fumarate & LC/MS polar & 1643 & $\underline{\mathrm{C} 00122}$ & HMDB00134 & 444972 & 0.71 & 1.04 & 0.69 \\
\hline & malate & LC/MS neg & 1303 & $\underline{\mathrm{C} 00149}$ & HMDB00156 & 525 & 0.76 & 0.97 & 0.78 \\
\hline & itaconate & LC/MS polar & 18373 & $\underline{\mathrm{C} 00490}$ & HMDB02092 & 811 & 0.92 & 0.59 & 1.58 \\
\hline & tricarballylate & LC/MS polar & 15729 & $\underline{\mathrm{C} 19806}$ & HMDB31193 & 14925 & 0.90 & 0.92 & 0.98 \\
\hline & 2-methylcitrate & LC/MS neg & 37483 & $\underline{\mathrm{CO} 2225}$ & HMDB00379 & 439681 & 1.09 & 1.03 & 1.06 \\
\hline & mesaconate (methylfumarate) & LC/MS polar & 18493 & $\underline{\mathrm{C} 01732}$ & HMDB00749 & 638129 & 0.91 & 0.62 & 1.46 \\
\hline \multirow{2}{*}{ Oxidative Phosphorylation } & acetylphosphate & LC/MS polar & 15488 & $\underline{\mathrm{C} 00227}$ & HMDB01494 & 186 & 1.13 & 0.71 & 1.60 \\
\hline & phosphate & LC/MS pos early & 42109 & $\underline{\mathrm{CO} 00009}$ & HMDB01429 & 1061 & 1.00 & 0.99 & 1.01 \\
\hline
\end{tabular}


bioRxiv preprint doi: https://doi.org/10.1101/2022.02.17.480875; this version posted February 21, 2022. The copyright holder for this preprint

(which was not certified by peer review) is the author/funder, who has granted bioRxiv a license to display the preprint in perpetuity. It is made available under aCC-BY-NC-ND 4.0 International license.

\begin{tabular}{|c|c|c|c|c|c|c|c|c|c|}
\hline Sub Pathway & Biochemical Name & Platform & Comp ID & KEGG & HMDB & PubChem & $\frac{\text { Gal4>UAS }}{\text { GAL4 Ctrl }}$ & $\frac{\text { Gal } 4>\mathrm{UAS}}{\text { UAS CtrI }}$ & $\underline{\text { UAS Ctrl }}$ \\
\hline Fatty Acid Synthesis & malonate & LC/MS polar & 15872 & $\underline{\mathrm{C} 00383}$ & HMDB00691 & 867 & 0.85 & 0.86 & 1.00 \\
\hline \multirow{3}{*}{ Medium Chain Fatty Acid } & caprate (10:0) & LC/MS neg & 1642 & $\underline{\mathrm{C} 01571}$ & HMDB00511 & 2969 & 1.45 & 1.04 & 1.40 \\
\hline & laurate (12:0) & LC/MS neg & 1645 & $\underline{\mathrm{C} 02679}$ & HMDB00638 & 3893 & 0.79 & 1.08 & 0.73 \\
\hline & 5-dodecenoate (12:1n7) & LC/MS neg & 33968 & & HMDB00529 & 5312378 & 1.17 & 1.14 & 1.03 \\
\hline \multirow{14}{*}{ Long Chain Fatty Acid } & myristate (14:0) & LC/MS neg & 1365 & $\underline{\mathrm{C} 06424}$ & HMDB00806 & 11005 & 1.18 & 1.04 & 1.14 \\
\hline & myristoleate (14:1n5) & LC/MS neg & 32418 & $\underline{\mathrm{C} 08322}$ & HMDB02000 & 5281119 & 0.99 & 1.38 & 0.72 \\
\hline & pentadecanoate (15:0) & LC/MS neg & 1361 & $\underline{C 16537}$ & HMDB00826 & 13849 & 1.40 & 0.98 & 1.43 \\
\hline & palmitate $(16: 0)$ & LC/MS neg & 1336 & $\underline{\mathrm{C} 00249}$ & HMDB00220 & 985 & 1.21 & 0.99 & 1.22 \\
\hline & palmitoleate (16:1n7) & LC/MS neg & 33447 & $\underline{\mathrm{C} 08362}$ & HMDB03229 & 445638 & 1.19 & 1.01 & 1.18 \\
\hline & margarate (17:0) & LC/MS neg & 1121 & & $\underline{\text { HMDB02259 }}$ & 10465 & 1.41 & 0.91 & 1.55 \\
\hline & 10-heptadecenoate (17:1n7) & LC/MS neg & 33971 & & HMDB60038 & 5312435 & 1.54 & 0.89 & 1.72 \\
\hline & stearate $(18: 0)$ & LC/MS neg & 1358 & C01530 & HMDB00827 & 5281 & 1.10 & 0.95 & 1.16 \\
\hline & oleate/vaccenate (18:1) & LC/MS neg & 52285 & & & & 1.16 & 1.04 & 1.11 \\
\hline & nonadecanoate (19:0) & LC/MS neg & 1356 & $\underline{C 16535}$ & HMDB00772 & 12591 & 1.25 & 1.08 & 1.16 \\
\hline & 10-nonadecenoate (19:1n9) & LC/MS neg & 33972 & & HMDB13622 & 5312513 & 1.16 & 0.72 & 1.62 \\
\hline & arachidate $(20: 0)$ & LC/MS neg & 1118 & $\underline{\mathrm{C} 06425}$ & HMDB02212 & 10467 & 1.34 & 0.92 & 1.45 \\
\hline & eicosenoate (20:1) & LC/MS neg & 33587 & $\underline{\mathrm{C} 16526}$ & HMDB02231 & 5282768 & 1.55 & 1.16 & 1.33 \\
\hline & erucate (22:1n9) & LC/MS neg & 1552 & $\underline{\mathrm{C} 08316}$ & HMDB02068 & 5281116 & 1.16 & 0.82 & 1.41 \\
\hline \multirow{8}{*}{ Polyunsaturated Fatty Acid (n3 and n6) } & hexadecatrienoate (16:3n3) & LC/MS neg & 57651 & & & 5312428 & 0.88 & 0.96 & 0.91 \\
\hline & stearidonate (18:4n3) & LC/MS neg & 33969 & $\underline{\mathrm{C} 16300}$ & HMDB06547 & 5312508 & 0.89 & 0.66 & 1.34 \\
\hline & eicosapentaenoate (EPA; 20:5n3) & LC/MS neg & 18467 & $\underline{\mathrm{C} 06428}$ & HMDB01999 & 446284 & 1.25 & 1.45 & 0.86 \\
\hline & linoleate $(18: 2 n 6)$ & LC/MS neg & 1105 & $\underline{\mathrm{C} 01595}$ & HMDB00673 & 5280450 & 1.10 & 0.96 & 1.14 \\
\hline & linolenate [alpha or gamma; (18:3n3 or 6)] & LC/MS neg & 34035 & $\underline{\mathrm{C} 06426}$ & HMDB03073 & 5280934 & 1.08 & 0.97 & 1.11 \\
\hline & dihomo-linolenate (20:3n3 or $n 6)$ & LC/MS neg & 35718 & $\underline{\mathrm{CO} 03242}$ & HMDB02925 & 5280581 & 0.80 & 0.87 & 0.92 \\
\hline & arachidonate $(20: 4 \mathrm{n} 6)$ & LC/MS neg & 1110 & $\underline{\mathrm{C} 00219}$ & HMDB01043 & 444899 & 1.30 & 1.19 & 1.10 \\
\hline & dihomo-linoleate (20:2n6) & LC/MS neg & 17805 & $\underline{\mathrm{C} 16525}$ & HMDB05060 & 6439848 & 1.61 & 1.87 & 0.86 \\
\hline \multirow{3}{*}{ Fatty Acid, Branched } & 13-methylmyristate (i15:0) & LC/MS neg & 38293 & & & 151014 & 1.20 & 0.86 & 1.40 \\
\hline & 15-methylpalmitate (i17:0) & LC/MS neg & 38768 & & & 17903417 & 1.18 & 0.77 & 1.54 \\
\hline & 17-methylstearate (i19:0) & LC/MS neg & 38296 & & HMDB37397 & 3083779 & 1.27 & 0.80 & 1.59 \\
\hline \multirow{11}{*}{ Fatty Acid, Dicarboxylate } & glutarate (C5-DC) & LC/MS polar & 396 & $\underline{\mathrm{C} 00489}$ & HMDB00661 & 743 & 0.90 & 1.00 & 0.90 \\
\hline & 2-hydroxyglutarate & LC/MS pos early & 37253 & $\underline{\mathrm{CO} 2630}$ & HMDB00606 & 43 & 1.10 & 1.04 & 1.06 \\
\hline & adipate (C6-DC) & LC/MS polar & 21134 & $\underline{\mathrm{C} 06104}$ & HMDB00448 & 196 & 0.90 & 1.38 & 0.65 \\
\hline & suberate (C8-DC) & LC/MS polar & 15730 & $\underline{\mathrm{C} 08278}$ & HMDB00893 & 10457 & 1.46 & 1.51 & 0.97 \\
\hline & azelate (C9-DC) & LC/MS neg & 18362 & $\underline{\mathrm{C} 08261}$ & HMDB00784 & 2266 & 2.01 & 2.14 & 0.94 \\
\hline & sebacate (C10-DC) & LC/MS polar & 32398 & $\mathrm{C} 08277$ & HMDB00792 & 5192 & 0.72 & 0.94 & 0.76 \\
\hline & dodecanedioate (C12-DC) & LC/MS neg & 32388 & $\underline{\mathrm{C} 02678}$ & HMDB00623 & 12736 & 0.59 & 0.94 & 0.62 \\
\hline & tetradecanedioate (C14-DC) & LC/MS neg & 35669 & & HMDB00872 & 13185 & 0.92 & 1.04 & 0.89 \\
\hline & hexadecanedioate (C16-DC) & LC/MS neg & 35678 & $\underline{\mathrm{C} 19615}$ & HMDB00672 & 10459 & 1.30 & 1.18 & 1.10 \\
\hline & hexadecenedioate $(\mathrm{C} 16: 1-\mathrm{DC})^{*}$ & LC/MS neg & 61862 & & & & 1.09 & 1.32 & 0.82 \\
\hline & ${\text { octadecadienedioate }(\mathrm{C} 18: 2-\mathrm{DC})^{\star}}^{*}$ & LC/MS neg & 61860 & & & & 1.00 & 1.00 & 1.00 \\
\hline \multirow{2}{*}{ Fatty Acid, Amino } & 2-aminooctanoate & LC/MS pos late & 43343 & & HMDB00991 & 69522 & 2.29 & 0.97 & 2.36 \\
\hline & $\mathrm{N}$-acetyl-2-aminooctanoate ${ }^{*}$ & LC/MS neg & 62059 & & HMDB59745 & 95555 & 1.15 & 0.90 & 1.28 \\
\hline \multirow{2}{*}{ Fatty Acid Metabolism (also BCAA Metabolism) } & propionylglycine & LC/MS polar & 31932 & & HMDB00783 & 98681 & 0.65 & 1.12 & 0.58 \\
\hline & methylmalonate (MMA) & LC/MS polar & 1496 & $\underline{\mathrm{C} 02170}$ & HMDB00202 & 487 & 0.70 & 0.96 & 0.73 \\
\hline Fatty Acid Metabolism(Acyl Glycine) & hexanoylglycine & LC/MS neg & 35436 & & HMDB00701 & 99463 & 1.19 & 1.10 & 1.08 \\
\hline \multirow{15}{*}{ Fatty Acid Metabolism(Acyl Carnitine) } & acetylcarnitine (C2) & LC/MS pos early & 32198 & $\underline{\mathrm{C} 02571}$ & HMDB00201 & 1 & 4.25 & 2.68 & 1.59 \\
\hline & myristoylcarnitine (C14) & LC/MS pos late & 33952 & & HMDB05066 & 6426854 & 28.81 & 26.39 & 1.09 \\
\hline & palmitoylcarnitine (C16) & LC/MS pos late & 44681 & $\underline{\mathrm{C} 02990}$ & HMDB00222 & 461 & 4.52 & 1.74 & 2.60 \\
\hline & palmitoleoylcarnitine $(\mathrm{C} 16: 1)^{\star}$ & LC/MS pos late & 53223 & & & 71464547 & 26.50 & 10.35 & 2.56 \\
\hline & stearoylcarnitine (C18) & LC/MS pos late & 34409 & & HMDB00848 & 6426855 & 1.43 & 1.06 & 1.34 \\
\hline & linoleoylcarnitine $(\mathrm{C} 18: 2)^{*}$ & LC/MS pos late & 46223 & & HMDB06469 & 6450015 & 6.77 & 3.14 & 2.15 \\
\hline & oleoylcarnitine (C18:1) & LC/MS pos late & 35160 & & HMDB05065 & 6441392 & 4.00 & 1.92 & 2.08 \\
\hline & arachidoylcarnitine $(\mathrm{C} 20)^{*}$ & LC/MS pos late & 57513 & & HMDB06460 & & 1.28 & 1.17 & 1.09 \\
\hline & \begin{tabular}{|l} 
behenoylcarnitine $(\mathrm{C} 22)^{\star}$ \\
\end{tabular} & LC/MS pos late & 57514 & & & & 1.31 & 1.09 & 1.20 \\
\hline & \begin{tabular}{|l} 
eicosenoylcarnitine $(\mathrm{C} 20: 1)^{\star}$ \\
\end{tabular} & LC/MS pos late & 57519 & & & & 2.28 & 1.24 & 1.84 \\
\hline & lignoceroylcarnitine (C24) ${ }^{*}$ & LC/MS pos late & 57515 & & & & 0.72 & 0.83 & 0.87 \\
\hline & margaroylcarnitine $(\mathrm{C} 17)^{*}$ & LC/MS pos late & 57512 & & HMDB06210 & & 4.05 & 1.47 & 2.76 \\
\hline & nervonoylcarnitine $(\mathrm{C} 24: 1)^{\star}$ & LC/MS pos late & 57531 & & & & 2.36 & 1.26 & 1.87 \\
\hline & cerotoylcarnitine $(\mathrm{C} 26)^{*}$ & LC/MS pos late & 57516 & & HMDB06347 & & 0.85 & 0.97 & 0.87 \\
\hline & ximenoylcarnitine $(\mathrm{C} 26: 1)^{\star}$ & LC/MS pos late & 57517 & & & & 0.75 & 0.88 & 0.85 \\
\hline \multirow{2}{*}{ Carnitine Metabolism } & deoxycarnitine & LC/MS pos early & 36747 & $\underline{\mathrm{C} 01181}$ & HMDB01161 & 134 & 1.77 & 1.17 & 1.52 \\
\hline & carnitine & LC/MS pos early & 15500 & $\underline{\mathrm{C} 00318}$ & HMDB00062 & 10917 & 1.62 & 1.01 & 1.59 \\
\hline
\end{tabular}


bioRxiv preprint doi: https://doi.org/10.1101/2022.02.17.480875; this version posted February 21, 2022. The copyright holder for this preprint

(which was not certified by peer review) is the author/funder, who has granted bioRxiv a license to display the preprint in perpetuity. It is made available under aCC-BY-NC-ND 4.0 International license.

\begin{tabular}{|c|c|c|c|c|c|c|c|c|c|}
\hline Sub Pathway & Biochemical Name & Platform & Comp ID & KEGG & HMDB & PubChem & $\frac{\text { Gal4>UAS }}{\text { GAL4 CtrI }}$ & $\frac{\text { Gal4>UAS }}{\text { UAS Ctrl }}$ & $\frac{\text { UAS Ctrl }}{\text { GAL4 Ctrl }}$ \\
\hline \multirow{7}{*}{ Fatty Acid, Monohydroxy } & 3-hydroxypropanoate & LC/MS polar & 1556 & $\underline{\underline{\mathrm{C} 01013}}$ & HMDB00700 & 68152 & 0.96 & 1.20 & 0.80 \\
\hline & 3-hydroxydecanoate & LC/MS neg & 22053 & & HMDB02203 & 26612 & 0.90 & 0.71 & 1.25 \\
\hline & 3-hydroxysebacate & LC/MS polar & 31943 & & HMDB00350 & 3017884 & 0.62 & 0.57 & 1.09 \\
\hline & 3-hydroxylaurate & LC/MS neg & 32457 & & HMDB00387 & 94216 & 1.07 & 1.30 & 0.82 \\
\hline & 3-hydroxymyristate & LC/MS neg & 21158 & & & 16064 & 1.63 & 1.02 & 1.59 \\
\hline & 3-hydroxyoleate ${ }^{*}$ & LCIMS neg & 61843 & & & & 1.51 & 0.88 & 1.72 \\
\hline & 13-HODE + 9-HODE & LCIMS neg & 37752 & & & 43013 & 1.19 & 1.05 & 1.13 \\
\hline \multirow{2}{*}{ Fatty Acid, Dihydroxy } & 12,13-DiHOME & LC/MS neg & 38395 & $\underline{\mathrm{C} 14829}$ & HMDB04705 & 10236635 & 0.63 & 0.99 & 0.63 \\
\hline & 9,10-DiHOME & LCIMS neg & 38399 & C14828 & HMDB04704 & 9966640 & 0.83 & 1.37 & 0.61 \\
\hline \multirow{4}{*}{ Endocannabinoid } & N-myristoyltaurine & LCIMS neg & 61825 & & & 3810823 & 1.24 & 0.63 & 1.97 \\
\hline & N-oleoyltaurine & LCIMS neg & 39732 & & & 6437033 & 1.16 & 0.81 & 1.42 \\
\hline & \begin{tabular}{|l} 
N-palmitoleoyltaurine \\
\end{tabular} & LCIMS neg & 61824 & & & & 0.97 & 0.82 & 1.18 \\
\hline & linoleoyl ethanolamide & LCIMS neg & 52608 & & HMDB12252 & 5283446 & 1.02 & 0.73 & 1.40 \\
\hline Inositol Metabolism & myo-inositol & LC/MS polar & 1124 & $\underline{\mathrm{C} 00137}$ & HMDB00211 & 892 & 1.03 & 0.94 & 1.10 \\
\hline \multirow{9}{*}{ Phospholipid Metabolism } & choline & LC/MS pos early & 15506 & $\underline{\mathrm{C} 00114}$ & HMDB00097 & 305 & 1.01 & 1.04 & 0.97 \\
\hline & choline phosphate & LC/MS pos early & 34396 & $\underline{\mathrm{C}} 00588$ & HMDB01565 & 1014 & 1.08 & 1.14 & 0.95 \\
\hline & cytidine 5 -diphosphocholine & LCIMS pos early & 34418 & $\underline{\mathrm{CO} 00307}$ & HMDB01413 & 13804 & 1.13 & 0.92 & 1.23 \\
\hline & glycerophosphorylcholine (GPC) & LC/MS pos early & 15990 & $\underline{\mathrm{C} 00670}$ & $\underline{\text { HMDB00086 }}$ & 71920 & 0.97 & 0.99 & 0.97 \\
\hline & phosphoethanolamine & LCIMS pos early & 1600 & $\underline{\mathrm{C} 00346}$ & HMDB00224 & 1015 & 0.93 & 1.05 & 0.88 \\
\hline & cytidine-5'-diphosphoethanolamine & LC/MS polar & 34410 & $\underline{\underline{c 00570}}$ & HMDB01564 & 123727 & 0.97 & 0.85 & 1.14 \\
\hline & glycerophosphoethanolamine & LC/MS polar & 37455 & $\underline{\mathrm{C} 01233}$ & HMDB00114 & 123874 & 0.89 & 0.97 & 0.91 \\
\hline & \begin{tabular}{|l|l} 
glycerophosphoserine* \\
\end{tabular} & LCIMS pos early & 57404 & & & 3081457 & 1.33 & 1.48 & 0.90 \\
\hline & \begin{tabular}{|l} 
glycerophosphoinositol* \\
\end{tabular} & LCIMS pos early & 52307 & & & 167572 & 1.23 & 1.31 & 0.93 \\
\hline \multirow{13}{*}{ Phosphatidylcholine (PC) } & 1-myristoyl-2-palmitoyl-GPC (14:0/16:0) & LC/MS pos late & 19258 & & HMDB07869 & 129657 & 0.79 & 0.92 & 0.87 \\
\hline & 1,2-dipalmitoyl-GPC (16:0/16:0) & LC/MS pos late & 19130 & & HMDB00564 & 452110 & 0.71 & 0.90 & 0.79 \\
\hline & 1-palmitoyl-2-palmitoleoyl-GPC (16:0/16:1)* & LC/MS pos late & 52470 & & HMDB07969 & & 1.04 & 0.92 & 1.12 \\
\hline & 1-palmitoyl-2-stearoyl-GPC (16:0/18:0) & LC/MS pos late & 52616 & & HMDB07970 & & 0.71 & 0.92 & 0.77 \\
\hline & 1-palmitoyl-2-oleoyl-GPC (16:0/18:1) & LC/MS pos late & 52461 & & HMDB07972 & 6436017 & 0.95 & 0.97 & 0.98 \\
\hline & 1,2-dipalmitoleoyl-GPC $(16: 1 / 16: 1)^{*}$ & LC/MS pos late & 52472 & & & & 0.95 & 0.83 & 1.15 \\
\hline & 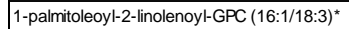 & LC/MS pos late & 53180 & & HMDB08008 & & 0.92 & 1.03 & 0.90 \\
\hline & 1,2-distearoyl-GPC (18:0/18:0) & LC/MS pos late & 19132 & & HMDB08036 & 94190 & 0.72 & 0.93 & 0.78 \\
\hline & \begin{tabular}{|l|} 
1-stearoyl-2-oleoyl-GPC (18:0/18:1) \\
\end{tabular} & LC/MS pos late & 52438 & & HMDB08038 & & 0.85 & 1.01 & 0.84 \\
\hline & 1-oleoyl-2-linoleoyl-GPC (18:1/18:2)* & LC/MS pos late & 52453 & & & & 0.90 & 0.88 & 1.02 \\
\hline & 1,2-dilinoleoyl-GPC (18:2/18:2) & LC/MS pos late & 52603 & & HMDB08138 & 5288075 & 0.78 & 0.81 & 0.97 \\
\hline & 1-linoleoyl-2-linolenoyl-GPC $(18: 2 / 18: 3)^{*}$ & LC/MS pos late & 53176 & & HMDB08141 & & 0.79 & 0.88 & 0.90 \\
\hline & \begin{tabular}{|l}
1,2 -dilinolenoyl-GPC $(18: 3 / 18: 3)^{*}$ \\
\end{tabular} & LC/MS pos late & 53179 & & HMDB08206 & & 0.77 & 0.89 & 0.87 \\
\hline \multirow{7}{*}{ Phosphatidylethanolamine (PE) } & 1,2-dipalmitoyl-GPE $(16: 0 / 16: 0)^{*}$ & LC/MS pos late & 57341 & & HMDB08923 & 445468 & 0.84 & 0.97 & 0.87 \\
\hline & 1-palmitoyl-2-stearoyl-GPE (16:0/18:0) & LC/MS pos late & 57388 & & HMDB08925 & 5326793 & 0.64 & 0.78 & 0.82 \\
\hline & 1-palmitoyl-2-oleoyl-GPE (16:0/18:1) & LC/MS pos late & 19263 & & $\underline{\mathrm{HMDB} 05320}$ & 5283496 & 0.96 & 0.94 & 1.02 \\
\hline & 1,2-dipalmitoleoyl-GPE $(16: 1 / 16: 1)^{*}$ & LC/MS pos late & 52688 & & HMDB05342 & 9546809 & 0.84 & 0.86 & 0.98 \\
\hline & 1-stearoyl-2-oleoyl-GPE (18:0/18:1) & LCMS pos late & 42448 & & HMDB08993 & & 0.96 & 1.03 & 0.93 \\
\hline & \begin{tabular}{|l} 
1-oleoyl-2-linoleoyl-GPE $(18: 1 / 18: 2)^{*}$ \\
\end{tabular} & LC/MS pos late & 52687 & & HMDB05349 & 9546753 & 0.93 & 0.91 & 1.03 \\
\hline & \begin{tabular}{|l} 
1,2-dilinoleoyl-GPE $(18: 2 / 18: 2)^{*}$ \\
\end{tabular} & LC/MS pos late & 53174 & & HMDB09093 & 9546812 & 0.85 & 0.85 & 0.99 \\
\hline Phosphatidylserine (PS) & 1,2-dioleoyl-GPS (18:1/18:1) & LC/MS pos late & 19191 & & & 6438639 & 1.29 & 0.95 & 1.35 \\
\hline \multirow{2}{*}{ Phosphatidylglycerol (PG) } & 1-palmitoyl-2-palmitoleoyl-GPG $(16: 0 / 16: 1)^{\star}$ & LC/MS pos late & 53213 & & & & 1.32 & 0.78 & 1.68 \\
\hline & 1-palmitoyl-2-oleoyl-GPG (16:0/18:1) & LC/MS pos late & 52448 & & & 5283509 & 0.91 & 0.81 & 1.11 \\
\hline \multirow{3}{*}{ Phosphatidylinositol (PI) } & 1,2-dipalmitoleoyl-GPI (16:1/16:1)* & LC/MS pos late & 52721 & & & & 1.06 & 0.90 & 1.18 \\
\hline & \begin{tabular}{|c|} 
1-palmitoyl-2-oleoyl-GPI (16:0/18:1)* \\
\end{tabular} & LC/MS polar & 52669 & & HMDB09783 & & 0.91 & 0.77 & 1.18 \\
\hline & 1-oleoyl-2-linoleoyl-GPI (18:1/18:2)* & LC/MS polar & 52451 & & HMDB09838 & & 0.89 & 0.90 & 0.99 \\
\hline
\end{tabular}


bioRxiv preprint doi: https://doi.org/10.1101/2022.02.17.480875; this version posted February 21, 2022. The copyright holder for this preprint (which was not certified by peer review) is the author/funder, who has granted bioRxiv a license to display the preprint in perpetuity. It is made available under aCC-BY-NC-ND 4.0 International license.

\begin{tabular}{|c|c|c|c|c|c|c|c|c|c|}
\hline Sub Pathway & Biochemical Name & Platform & Comp ID & KEGG & HMDB & PubChem & $\frac{\mathrm{Gal} 4>\mathrm{UAS}}{\mathrm{GAL4} \mathrm{Ctrl}}$ & $\frac{\text { Gal } 4>\text { UAS }}{\text { UAS CtrI }}$ & $\frac{\text { UAS Ctrl }}{\text { GAL4 CtrI }}$ \\
\hline \multirow{26}{*}{ Lysophospholipid } & 1-linolenoyl-GPG $(18: 3)^{\star}$ & LC/MS neg & 62368 & & & & 1.17 & 0.98 & 1.20 \\
\hline & 1-palmitoyl-GPC (16:0) & LC/MS pos late & 33955 & & HMDB10382 & 86554 & 0.79 & 0.98 & 0.81 \\
\hline & 2-palmitoyl-GPC $(16: 0)^{*}$ & LC/MS pos late & 35253 & & HMDB61702 & 15061532 & 0.98 & 0.92 & 1.07 \\
\hline & 1-palmitoleoyl-GPC $(16: 1)^{\star}$ & LC/MS pos late & 33230 & & HMDB10383 & 24779461 & 1.06 & 1.07 & 0.99 \\
\hline & 2-palmitoleoyl-GPC $(16: 1)^{\star}$ & LC/MS pos late & 35819 & & HMDB10383 & & 1.14 & 0.99 & 1.15 \\
\hline & 1-stearoyI-GPC (18:0) & LC/MS pos late & 33961 & & HMDB10384 & 497299 & 0.75 & 1.01 & 0.75 \\
\hline & 1-oleoyl-GPC (18:1) & LC/MS pos late & 48258 & & $\underline{\text { HMDB02815 }}$ & 16081932 & 1.06 & 1.07 & 0.99 \\
\hline & 1-linoleoyl-GPC (18:2) & LC/MS neg & 34419 & $\underline{\mathrm{C}} 04100$ & HMDB10386 & 11988421 & 1.15 & 0.95 & 1.20 \\
\hline & 1-linolenoyl-GPC $(18: 3)^{*}$ & LC/MS pos late & 45951 & & HMDB10388 & & 0.74 & 0.98 & 0.75 \\
\hline & 1-palmitoyl-GPE (16:0) & LC/MS pos late & 35631 & & HMDB11503 & 9547069 & 0.85 & 0.93 & 0.92 \\
\hline & 1-stearoyl-GPE (18:0) & LC/MS pos late & 42398 & & HMDB11130 & 9547068 & 0.76 & 0.91 & 0.84 \\
\hline & 2-stearoyl-GPE (18:0)* & LC/MS neg & 41220 & & HMDB11129 & & 0.90 & 1.05 & 0.86 \\
\hline & 1-oleoyl-GPE (18:1) & LC/MS pos late & 35628 & & HMDB11506 & 9547071 & 0.98 & 0.94 & 1.04 \\
\hline & 1-linoleoyl-GPE (18:2)* & LC/MS pos late & 36600 & & HMDB11507 & 52925130 & 0.95 & 1.00 & 0.95 \\
\hline & 1-palmitoyl-GPS $(16: 0)^{*}$ & LC/MS neg & 46130 & & & 9547100 & 1.57 & 1.17 & 1.35 \\
\hline & 1-stearoyl-GPS $(18: 0)^{*}$ & LC/MS neg & 45966 & & & 9547101 & 1.23 & 0.87 & 1.42 \\
\hline & 1-oleoyl-GPS (18:1) & LC/MS neg & 19260 & & HMDB61694 & 9547099 & 1.49 & 1.00 & 1.49 \\
\hline & 1-linoleoyl-GPS (18:2)* & LC/MS neg & 43676 & & & & 1.11 & 0.81 & 1.38 \\
\hline & 1-palmitoyl-GPG (16:0)* & LC/MS neg & 45970 & & & 3300276 & 1.03 & 0.77 & 1.34 \\
\hline & 1-stearoyI-GPG (18:0) & LC/MS neg & 34437 & & & & 0.96 & 0.93 & 1.03 \\
\hline & 1-oleoyI-GPG $(18: 1)^{\star}$ & LC/MS neg & 45968 & & & & 1.27 & 1.16 & 1.09 \\
\hline & 1-linoleoyl-GPG (18:2)* & LC/MS neg & 54885 & & & & 1.11 & 0.91 & 1.22 \\
\hline & 1-palmitoyl-GPI (16:0) & LC/MS neg & 35305 & & $\underline{\text { HMDB61695 }}$ & & 1.11 & 0.95 & 1.17 \\
\hline & 1-stearoyl-GPI (18:0) & LC/MS neg & 19324 & & HMDB61696 & & 0.83 & 0.66 & 1.26 \\
\hline & 1-oleoyl-GPI $(18: 1)^{\star}$ & LC/MS neg & 36602 & & & & 1.65 & 1.12 & 1.48 \\
\hline & 1-linoleoyI-GPI $(18: 2)^{\star}$ & LC/MS neg & 36594 & & & & 1.20 & 0.99 & 1.21 \\
\hline Glycolipid Metabolism & 1,2-dilinoleoyl-galactosylglycerol (18:2/18:2)* & LC/MS pos late & 54899 & & & 6535011 & 1.05 & 0.90 & 1.16 \\
\hline \multirow{3}{*}{ Plasmalogen } & 1-(1-enyl-palmitoyl)-2-oleoyl-GPE (P-16:0/18:1)* & LC/MS pos late & 52477 & & HMDB11342 & & 1.23 & 1.06 & 1.15 \\
\hline & 1-(1-enyl-stearoyl)-2-oleoyl-GPE (P-18:0/18:1) & LC/MS pos late & 52614 & & $\underline{\text { HMDB11375 }}$ & & 0.94 & 0.86 & 1.09 \\
\hline & 1-(1-enyl-stearoyl)-2-linoleoyl-GPE (P-18:0/18:2)* & LC/MS pos late & 52748 & & HMDB11376 & & 0.75 & 1.02 & 0.74 \\
\hline Lysoplasmalogen & 1-(1-enyl-stearoyl)-GPE (P-18:0)* & LC/MS pos late & 39271 & & & & 0.67 & 1.01 & 0.67 \\
\hline \multirow{3}{*}{ Glycerolipid Metabolism } & glycerol & LC/MS neg & 15122 & $\underline{\mathrm{C} 00116}$ & $\underline{\mathrm{HMDB} 00131}$ & 753 & 1.19 & 1.09 & 1.09 \\
\hline & glycerol 3-phosphate & LC/MS polar & 43847 & $\underline{\mathrm{C} 000993}$ & $\underline{\mathrm{HMDB} 00126}$ & 754 & 1.09 & 1.15 & 0.95 \\
\hline & glycerophosphoglycerol & LC/MS polar & 48857 & $\mathrm{C} 03274$ & & 439964 & 1.02 & 1.26 & 0.81 \\
\hline \multirow{10}{*}{ Monoacylglycerol } & 1-myristoylglycerol (14:0) & LC/MS neg & 35625 & C01885 & HMDB11561 & 79050 & 1.26 & 0.76 & 1.65 \\
\hline & 1-palmitoylglycerol (16:0) & LC/MS neg & 21127 & & HMDB31074 & 14900 & 1.04 & 0.93 & 1.12 \\
\hline & 1-palmitoleoylglycerol $(16: 1)^{*}$ & LC/MS neg & 52431 & & HMDB11565 & & 2.31 & 1.29 & 1.79 \\
\hline & 1-oleoylglycerol (18:1) & LC/MS neg & 21184 & & $\underline{\text { HMDB11567 }}$ & 5283468 & 1.85 & 1.31 & 1.41 \\
\hline & 1-linoleoylglycerol (18:2) & LC/MS neg & 27447 & & & 5283469 & 1.95 & 1.40 & 1.40 \\
\hline & 2-myristoylglycerol (14:0) & LC/MS neg & 34383 & & HMDB11530 & 137938 & 1.06 & 0.75 & 1.41 \\
\hline & 2-palmitoylglycerol (16:0) & LC/MS neg & 33419 & & HMDB11533 & 123409 & 1.49 & 1.09 & 1.37 \\
\hline & 2-palmitoleoylglycerol $(16: 1)^{*}$ & LC/MS neg & 52432 & & $\underline{\text { HMDB11565 }}$ & & 2.25 & 1.15 & 1.96 \\
\hline & 2-oleoylglycerol (18:1) & LC/MS neg & 21232 & & $\underline{\mathrm{HMDB} 11537}$ & 5319879 & 1.66 & 0.87 & 1.90 \\
\hline & 2-linoleoylglycerol (18:2) & LC/MS neg & 32506 & & HMDB11538 & 5365676 & 1.61 & 1.64 & 0.98 \\
\hline \multirow{11}{*}{ Diacylglycerol } & diacylglycerol (12:0/18:1, 14:0/16:1, 16:0/14:1) [2] & LC/MS pos late & 55001 & & & & 1.23 & 1.19 & 1.03 \\
\hline & diacylglycerol (14:0/18:1, 16:0/16:1) [2] & LC/MS pos late & 54954 & & & & 1.39 & 1.07 & 1.30 \\
\hline & diacylglycerol (16:1/18:2 [2], 16:0/18:3 [1]) & LC/MS pos late & 54966 & & & & 1.18 & 1.10 & 1.07 \\
\hline & palmitoyl-oleoyl-glycerol (16:0/18:1) [2] $]^{*}$ & LC/MS pos late & 54942 & C13861 & HMDB07102 & & 1.45 & 0.93 & 1.56 \\
\hline & palmitoyl-linoleoyl-glycerol (16:0/18:2) [2] ${ }^{\star}$ & LC/MS pos late & 52634 & & $\underline{\text { HMDB07103 }}$ & & 1.55 & 0.93 & 1.67 \\
\hline & palmitoleoyl-oleoyl-glycerol (16:1/18:1) [2] & LC/MS pos late & 52631 & & & & 1.12 & 1.07 & 1.04 \\
\hline & palmitoleoyl-linoleoyl-glycerol (16:1/18:2) [1] & LC/MS pos late & 54967 & & HMDB07132 & & 1.22 & 1.13 & 1.08 \\
\hline & oleoyl-oleoyl-glycerol (18:1/18:1) [2] ${ }^{*}$ & LC/MS pos late & 54946 & & $\underline{\text { HMDB07218 }}$ & & 1.08 & 1.04 & 1.03 \\
\hline & oleoyl-linoleoyl-glycerol (18:1/18:2) [2] & LC/MS pos late & 46799 & & $\underline{\mathrm{HMDB} 07219}$ & & 1.00 & 1.17 & 0.86 \\
\hline & oleoyl-linolenoyl-glycerol (18:1/18:3) [2] ${ }^{\star}$ & LC/MS pos late & 54970 & & $\underline{\text { HMDB07220 }}$ & & 0.93 & 1.81 & 0.51 \\
\hline & linoleoyl-linolenoyl-glycerol (18:2/18:3) [2] & LC/MS pos late & 54964 & & $\underline{\mathrm{HMDB} 07250}$ & & 0.70 & 1.22 & 0.57 \\
\hline
\end{tabular}


bioRxiv preprint doi: https://doi.org/10.1101/2022.02.17.480875; this version posted February 21, 2022. The copyright holder for this preprint

(which was not certified by peer review) is the author/funder, who has granted bioRxiv a license to display the preprint in perpetuity. It is made available under aCC-BY-NC-ND 4.0 International license.

\begin{tabular}{|c|c|c|c|c|c|c|c|c|c|}
\hline Sub Pathway & Biochemical Name & Platform & Comp ID & KEGG & HMDB & PubChem & $\frac{\text { Gal4>UAS }}{\text { GAL4 Ctrl }}$ & $\frac{\text { Gal4>UAS }}{\text { UAS Ctrl }}$ & $\frac{\text { UAS Ctrl }}{\text { GAL4 Ctrl }}$ \\
\hline \multirow{3}{*}{ Sphingolipid Synthesis } & sphinganine & LC/MS pos late & 17769 & $\underline{\mathrm{C} 00836}$ & HMDB00269 & 3126 & 0.55 & 1.00 & 0.55 \\
\hline & tetradecasphinganine $(\mathrm{d} 14: 0)^{\star}$ & LC/MS pos late & 57543 & & & & 1.15 & 1.08 & 1.07 \\
\hline & hexadecasphinganine $(\mathrm{d} 16: 0)^{\star}$ & LC/MS pos late & 57544 & C13915 & & 656816 & 1.21 & 1.04 & 1.16 \\
\hline \multirow{2}{*}{ Dihydroceramides } & $\mathrm{N}$-arachidoyl-tetradecanoylsphinganine (d14:0/20:0) & LC/MS pos late & 57499 & & & & 0.89 & 1.07 & 0.84 \\
\hline & $\mathrm{N}$-behenoyl-tetradecanoylsphinganine $(\mathrm{d} 14: 0 / 22: 0)^{\star}$ & LC/MS pos late & 57501 & & & & 0.81 & 0.94 & 0.86 \\
\hline \multirow{3}{*}{ Ceramides } & N-stearoyl-tetradecanoylsphingosine (d14:1/18:0)* & LC/MS pos late & 57500 & & & & 1.02 & 1.09 & 0.94 \\
\hline & $\mathrm{N}$-arachidoyl-tetradecanoylsphingosine (d14:1/20:0) & LC/MS pos late & 57505 & & & & 0.95 & 1.17 & 0.81 \\
\hline & N-behenoyl-tetradecanoylsphingosine $(\mathrm{d} 14: 1 / 22: 0)^{\star}$ & LC/MS pos late & 57494 & & & & 0.94 & 0.97 & 0.97 \\
\hline \multirow{3}{*}{ Hexosylceramides (HCER) } & glycosyl-N-arachidoyl-tetradecanoylsphingosine (d1 & LC/MS pos late & 57496 & & & & 1.33 & 1.04 & 1.28 \\
\hline & glycosyl-N-behenoyl-tetradecasphingosine (d14:1/2 & LC/MS pos late & 57849 & & & & 2.56 & 0.90 & 2.84 \\
\hline & glycosyl ceramide (d14:1/24:0, d16:1/22:0)* & LC/MS pos late & 57495 & & & & 5.01 & 0.70 & 7.14 \\
\hline Lactosylceramides (LCER) & lactosyl- $\mathrm{N}$-arachidoyl-tetradecanoylsphingosine (d14 & LC/MS pos late & 57498 & & & & 1.44 & 1.44 & 1.00 \\
\hline \multirow{2}{*}{ Sphingosines } & tetradecanoylsphingosine $(\mathrm{d} 14: 1)^{\star}$ & LC/MS pos late & 57493 & & & & 1.15 & 0.98 & 1.17 \\
\hline & hexadecasphingosine $(\mathrm{d} 16: 1)^{\star}$ & LC/MS pos late & 57428 & & & & 1.04 & 1.03 & 1.01 \\
\hline \multirow{2}{*}{ Mevalonate Metabolism } & 3-hydroxy-3-methylglutarate & LC/MS polar & 531 & $\underline{\mathrm{C} 03761}$ & HMDB00355 & 1662 & 1.07 & 1.08 & 1.00 \\
\hline & mevalonate & LC/MS polar & 39583 & $\underline{\mathrm{CO} 2104}$ & HMDB00227 & 439230 & 0.89 & 1.91 & 0.46 \\
\hline \multirow{3}{*}{ Sterol } & beta-sitosterol & LC/MS pos late & 27414 & $\underline{\mathrm{C} 01753}$ & HMDB00852 & 222284 & 1.08 & 1.14 & 0.94 \\
\hline & campesterol & LC/MS pos late & 33997 & $\underline{\mathrm{C} 01789}$ & HMDB02869 & 173183 & 1.00 & 1.19 & 0.84 \\
\hline & ergosterol & LC/MS pos late & 27553 & $\underline{\mathrm{C} 01694}$ & HMDB00878 & 444679 & 0.93 & 0.99 & 0.94 \\
\hline \multirow{11}{*}{ Purine Metabolism, (Hypo)Xanthine/lnosine containin } & inosine 5'-monophosphate (IMP) & LC/MS pos early & 2133 & $\underline{\mathrm{C} 00130}$ & HMDB00175 & 8582 & 1.19 & 0.70 & 1.70 \\
\hline & inosine & LC/MS neg & 1123 & $\underline{\mathrm{C} 00294}$ & HMDB00195 & 6021 & 0.95 & 1.02 & 0.93 \\
\hline & hypoxanthine & LC/MS polar & 3127 & $\underline{\mathrm{C} 00262}$ & $\underline{\mathrm{HMDB} 00157}$ & 790 & 0.54 & 1.17 & 0.46 \\
\hline & xanthine & LC/MS polar & 3147 & $\underline{\mathrm{C} 00385}$ & HMDB00292 & 1188 & 0.98 & 1.55 & 0.63 \\
\hline & \begin{tabular}{|l|} 
xanthosine 5'-monophosphate (xmp) \\
\end{tabular} & LC/MS neg & 12024 & $\underline{\mathrm{CO} 00655}$ & $\underline{\mathrm{HMDB} 01554}$ & 73323 & 0.75 & 1.00 & 0.75 \\
\hline & gxanthosine & LC/MS neg & 15136 & $\underline{\mathrm{C} 01762}$ & HMDB00299 & 64959 & 1.69 & 2.14 & 0.79 \\
\hline & N1-methylinosine & LC/MS pos early & 48351 & & $\underline{\mathrm{HMDB} 02721}$ & 65095 & 2.71 & 1.36 & 2.00 \\
\hline & 2'-deoxyinosine & LC/MS neg & 15076 & $\underline{\mathrm{C} 05512}$ & HMDB00071 & 65058 & 2.82 & 2.51 & 1.12 \\
\hline & urate & LC/MS neg & 1604 & $\underline{\mathrm{C} 00366}$ & HMDB00289 & 1175 & 0.73 & 0.98 & 0.74 \\
\hline & uric acid ribonucleoside* & LC/MS neg & 62102 & & & 164933 & 1.08 & 1.56 & 0.70 \\
\hline & allantoin & LC/MS polar & 1107 & $\underline{\mathrm{C} 02350}$ & HMDB00462 & 204 & 2.88 & 0.93 & 3.09 \\
\hline \multirow{9}{*}{ Purine Metabolism, Adenine containing } & adenosine 5'-diphosphate (ADP) & LC/MS neg & 3108 & $\underline{\mathrm{CO} 00008}$ & $\underline{\mathrm{HMDB} 01341}$ & 6022 & 1.08 & 0.56 & 1.93 \\
\hline & adenosine 5'-monophosphate (AMP) & LC/MS pos early & 32342 & $\underline{\mathrm{CO00020}}$ & $\underline{\mathrm{HMDB} 00045}$ & 6083 & 1.13 & 0.65 & 1.74 \\
\hline & adenosine $3^{\prime}$-monophosphate (3'-AMP) & LC/MS neg & 35142 & $\underline{\mathrm{C} 01367}$ & HMDB03540 & 41211 & 0.76 & 0.75 & 1.00 \\
\hline & \begin{tabular}{|l|} 
adenosine-2',3'-cyclic monophosphate \\
\end{tabular} & LC/MS neg & 37467 & $\underline{\mathrm{CO} 2353}$ & $\underline{\mathrm{HMDB} 11616}$ & 2024 & 1.01 & 0.87 & 1.17 \\
\hline & adenosine & LC/MS pos early & 555 & $\underline{\mathrm{C} 00212}$ & HMDB00050 & 60961 & 1.00 & 0.98 & 1.02 \\
\hline & adenine & LC/MS pos early & 554 & $\underline{\mathrm{C} 00147}$ & $\underline{\mathrm{HMDB} 00034}$ & 190 & 0.59 & 0.85 & 0.70 \\
\hline & 1-methyladenine & LC/MS pos early & 1527 & $\underline{\mathrm{C} 02216}$ & HMDB11599 & 78821 & 0.46 & 1.11 & 0.41 \\
\hline & N1-methyladenosine & LC/MS pos early & 15650 & $\underline{\mathrm{CO} 2494}$ & $\underline{\mathrm{HMDB} 03331}$ & 27476 & 0.87 & 1.03 & 0.85 \\
\hline & N6-succinyladenosine & LC/MS pos early & 48130 & & HMDB00912 & 165243 & 0.96 & 0.72 & 1.32 \\
\hline \multirow{10}{*}{ Purine Metabolism, Guanine containing } & guanosine 5'- diphosphate (GDP) & LC/MS neg & 2848 & $\underline{\mathrm{CO00035}}$ & $\underline{\mathrm{HMDB} 01201}$ & 8977 & 0.80 & 0.69 & 1.16 \\
\hline & guanosine 5'- monophosphate (5'-GMP) & LC/MS neg & 2849 & $\underline{\mathrm{CO} 00144}$ & $\underline{\mathrm{HMDB} 01397}$ & 6804 & 0.98 & 0.75 & 1.31 \\
\hline & guanosine-2',3'-cyclic monophosphate & LC/MS neg & 37139 & C06194 & HMDB11629 & 92823 & 1.01 & 0.75 & 1.35 \\
\hline & guanosine & LC/MS neg & 1573 & $\underline{\mathrm{CO} 00387}$ & HMDB00133 & 6802 & 1.26 & 1.18 & 1.07 \\
\hline & guanine & LC/MS pos early & 32352 & $\underline{\mathrm{C} 00242}$ & HMDB00132 & 764 & 1.05 & 0.99 & 1.06 \\
\hline & 7-methylguanine & LC/MS pos early & 35114 & $\underline{\mathrm{CO} 02242}$ & $\underline{\mathrm{HMDB} 00897}$ & 11361 & 0.99 & 1.20 & 0.82 \\
\hline & 2'-O-methylguanosine & LC/MS neg & 36811 & $\underline{\mathrm{CO} 4545}$ & & & 1.53 & 1.18 & 1.30 \\
\hline & 7-methylguanosine & LC/MS pos early & 31580 & $\underline{\mathrm{C} 20674}$ & & & 1.15 & 0.91 & 1.26 \\
\hline & N2,N2-dimethylguanosine & LC/MS neg & 35137 & & HMDB04824 & 92919 & 1.86 & 1.11 & 1.68 \\
\hline & 2'-deoxyguanosine & LC/MS neg & 1411 & C00330 & HMDB00085 & 187790 & 1.36 & 1.22 & 1.12 \\
\hline
\end{tabular}


bioRxiv preprint doi: https://doi.org/10.1101/2022.02.17.480875; this version posted February 21, 2022. The copyright holder for this preprint

(which was not certified by peer review) is the author/funder, who has granted bioRxiv a license to display the preprint in perpetuity. It is made available under aCC-BY-NC-ND 4.0 International license.

\begin{tabular}{|c|c|c|c|c|c|c|c|c|c|}
\hline Sub Pathway & Biochem ical Name & Platform & Comp ID & KEGG & HMDB & PubChem & $\frac{\text { Gal4>UAS }}{\text { GAL4 Ctrl }}$ & $\frac{\text { Gal } 4>\text { UAS }}{\text { UAS Ctrl }}$ & $\underline{\text { UAS Ctrl }}$ \\
\hline \multirow{2}{*}{ Pyrimidine Metabolism, Orotate containing } & dihydroorotate & LC/MS polar & 601 & $\underline{\mathrm{C} 00337}$ & HMDB03349 & 648 & 4.52 & 0.68 & 6.61 \\
\hline & orotate & LC/MS polar & 1505 & $\underline{\mathrm{C} 00295}$ & HMDB00226 & 967 & 1.34 & 0.92 & 1.45 \\
\hline \multirow{8}{*}{ Pyrimidine Metabolism, Uracil containing } & uridine-2',3'-cyclic monophosphate & LC/MS neg & 37137 & $\mathrm{C} 02355$ & HMDB11640 & 439715 & 0.78 & 0.79 & 0.98 \\
\hline & uridine & LC/MS neg & 606 & $\underline{\mathrm{C} 00299}$ & $\underline{\mathrm{HMDB} 00296}$ & 6029 & 1.50 & 1.53 & 0.98 \\
\hline & uracil & LC/MS polar & 605 & $\underline{\mathrm{C} 00106}$ & HMDB00300 & 1174 & 0.90 & 0.91 & 1.00 \\
\hline & pseudouridine & LC/MS neg & 33442 & $\underline{\mathrm{C} 02067}$ & $\underline{\mathrm{HMDB} 00767}$ & 15047 & 1.49 & 1.74 & 0.85 \\
\hline & 2'-O-methyluridine & LC/MS neg & 57655 & & & 102212 & 1.69 & 2.76 & 0.61 \\
\hline & 3-ureidopropionate & LC/MS pos early & 3155 & $\mathrm{C} 02642$ & HMDB00026 & 111 & 1.00 & 1.29 & 0.77 \\
\hline & beta-alanine & LC/MS pos early & 55 & $\underline{\mathrm{C} 00099}$ & HMDB00056 & 239 & 1.21 & 1.00 & 1.21 \\
\hline & $\mathrm{N}$-acetyl-beta-alanine & LC/MS polar & 37432 & $\underline{\mathrm{C} 01073}$ & & 76406 & 1.07 & 1.20 & 0.90 \\
\hline \multirow{8}{*}{ Pyrimidine Metabolism, Cytidine containing } & cytidine 5'-monophosphate (5'-CMP) & LC/MS pos early & 2372 & $\underline{\mathrm{C} 00055}$ & HMDB00095 & 6131 & 1.55 & 1.00 & 1.55 \\
\hline & cytidine 2' or 3'-monophosphate (2' or 3'-CMP) & LC/MS pos early & 61705 & & & & 0.96 & 0.93 & 1.03 \\
\hline & cytidine 2',3'-cyclic monophosphate & LC/MS neg & 37465 & $\mathrm{C} 02354$ & HMDB11691 & 417654 & 0.76 & 0.82 & 0.92 \\
\hline & cytidine & LC/MS neg & 514 & $\underline{\mathrm{C} 00475}$ & HMDB00089 & 6175 & 1.82 & 1.47 & 1.24 \\
\hline & cytosine & LC/MS pos early & 573 & $\underline{\mathrm{C} 00380}$ & HMDB00630 & 597 & 1.05 & 1.39 & 0.76 \\
\hline & 3-methylcytidine & LC/MS pos early & 35132 & & & 159649 & 1.28 & 1.21 & 1.06 \\
\hline & 5-methylcytidine & LC/MS pos early & 22119 & & HMDB00982 & 92918 & 2.98 & 1.09 & 2.72 \\
\hline & 2'-O-methylcytidine & LC/MS pos early & 57554 & & & 150971 & 2.78 & 1.94 & 1.43 \\
\hline Pyrimidine Metabolism, Thymine containing & 3-aminoisobutyrate & LC/MS pos early & 1566 & $\underline{\mathrm{C} 05145}$ & HMDB03911 & 64956 & 0.56 & 0.96 & 0.59 \\
\hline Purine and Pyrimidine Metabolism & methylphosphate & LC/MS pos early & 37070 & & HMDB61711 & 13130 & 1.17 & 0.94 & 1.24 \\
\hline Dinucleotide & $\left(3^{\prime}-5^{\prime}\right)$-adenylyluridine & LC/MS neg & 52740 & & & 112074 & 1.00 & 1.00 & 1.00 \\
\hline \multirow{6}{*}{ Nicotinate and Nicotinamide Metabolism } & nicotinate & LC/MS pos early & 1504 & $\underline{\mathrm{C} 00253}$ & HMDB01488 & 938 & 0.93 & 1.21 & 0.77 \\
\hline & nicotinamide ribonucleotide (NMN) & LC/MS pos early & 22152 & $\underline{\mathrm{C} 00455}$ & HMDB00229 & 14180 & 1.11 & 1.05 & 1.06 \\
\hline & nicotinamide riboside & LC/MS pos early & 33013 & $\underline{\mathrm{C} 03150}$ & HMDB00855 & 439924 & 1.37 & 2.01 & 0.68 \\
\hline & nicotinamide adenine dinucleotide (NAD+) & LC/MS pos early & 5278 & $\underline{\mathrm{C} 00003}$ & HMDB00902 & 5893 & 0.90 & 0.76 & 1.19 \\
\hline & nicotinate adenine dinucleotide (NAAD+) & LC/MS neg & 15725 & & & 25246170 & 0.88 & 0.75 & 1.18 \\
\hline & trigonelline ( $\mathrm{N}$-methylnicotinate) & LC/MS pos early & 32401 & $\underline{\mathrm{C} 01004}$ & HMDB00875 & 5570 & 1.11 & 0.90 & 1.23 \\
\hline \multirow{2}{*}{ Riboflavin Metabolism } & riboflavin (Vitamin B2) & LC/MS pos early & 1827 & $\underline{\mathrm{C} 00255}$ & HMDB00244 & 493570 & 2.72 & 0.71 & 3.84 \\
\hline & flavin adenine dinucleotide (FAD) & LC/MS neg & 2134 & $\underline{\mathrm{C} 00016}$ & HMDB01248 & 643975 & 0.73 & 0.74 & 0.99 \\
\hline \multirow{2}{*}{ Pantothenate and CoA Metabolism } & pantothenate & LC/MS neg & 1508 & $\underline{\mathrm{C} 00864}$ & HMDB00210 & 6613 & 0.75 & 0.91 & 0.82 \\
\hline & pantetheine & LC/MS pos early & 57555 & $\mathrm{C} 00831$ & & 439322 & 0.52 & 0.75 & 0.70 \\
\hline \multirow{4}{*}{ Ascorbate and Aldarate Metabolism } & ascorbate (Vitamin C) & LC/MS pos early & 32354 & $\underline{\mathrm{C} 00072}$ & HMDB00044 & & 1.63 & 1.24 & 1.31 \\
\hline & dehydroascorbate & LC/MS polar & 1659 & $\underline{\mathrm{C} 05422}$ & HMDB01264 & 835 & 0.58 & 0.70 & 0.82 \\
\hline & threonate & LC/MS polar & 27738 & $\mathrm{C} 01620$ & HMDB00943 & 151152 & 0.97 & 1.11 & 0.87 \\
\hline & gulonate $^{*}$ & LC/MS polar & 46957 & $\underline{\mathrm{C} 00257}$ & HMDB03290 & 9794176 & 0.75 & 1.01 & 0.74 \\
\hline \multirow{2}{*}{ Tocopherol Metabolism } & alpha-tocopherol & LC/MS pos late & 1561 & $\underline{\mathrm{C} 02477}$ & $\underline{\mathrm{HMDB} 01893}$ & 14985 & 1.09 & 1.23 & 0.89 \\
\hline & gamma-tocopherol/beta-tocopherol & LC/MS pos late & 52473 & & & & 1.58 & 1.51 & 1.04 \\
\hline Biotin Metabolism & biotin & LC/MS pos early & 568 & $\mathrm{C} 00120$ & HMDB00030 & 171548 & 1.31 & 1.20 & 1.09 \\
\hline \multirow{2}{*}{ Tetrahydrobiopterin Metabolism } & biopterin & LC/MS neg & 12358 & $\underline{\mathrm{C} 06313}$ & $\underline{\mathrm{HMDB} 00468}$ & 445040 & 0.67 & 0.85 & 0.79 \\
\hline & dihydrobiopterin & LC/MS pos early & 35129 & $\mathrm{C} 00268$ & HMDB00038 & 1879 & 0.52 & 0.78 & 0.66 \\
\hline \multirow{4}{*}{ Pterin Metabolism } & isoxanthopterin & LC/MS pos early & 27732 & $\underline{\mathrm{C} 03975}$ & HMDB00704 & 10729 & 0.82 & 0.76 & 1.09 \\
\hline & pterin & LC/MS neg & 43023 & $\mathrm{C} 00715$ & HMDB00802 & 73000 & 1.23 & 1.12 & 1.09 \\
\hline & sepiapterin & LC/MS pos early & 48139 & $\underline{\mathrm{C} 00835}$ & HMDB00238 & 65253 & 0.54 & 0.83 & 0.65 \\
\hline & xanthopterin & LC/MS polar & 54728 & & & 8397 & 0.88 & 0.70 & 1.26 \\
\hline Hemoglobin and Porphyrin Metabolism & 5-aminolevulinate & LC/MS pos early & 2290 & $\underline{\mathrm{C} 00430}$ & HMDB01149 & 137 & 0.72 & 1.67 & 0.43 \\
\hline Thiamine Metabolism & thiamin (Vitamin B1) & LC/MS pos early & 5341 & $\underline{\mathrm{C} 00378}$ & $\underline{\mathrm{HMDB} 00235}$ & 1130 & 0.61 & 0.56 & 1.09 \\
\hline miamine ivelaboism & thiamin monophosphate & LC/MS pos early & 15798 & $\underline{\mathrm{C} 01081}$ & $\underline{\mathrm{HMDB} 02666}$ & 3382778 & 0.92 & 0.61 & 1.51 \\
\hline & carotene diol (1) & LC/MS pos late & 57635 & & & & 0.88 & 1.10 & 0.80 \\
\hline Vitamin A Metabolism & carotene diol (2) & LC/MS pos late & 57636 & & & & 0.78 & 1.25 & 0.62 \\
\hline & carotene diol (3) & LC/MS pos late & 57637 & & & & 0.52 & 1.09 & 0.48 \\
\hline Vitamin B6 Metabolism & pyridoxal & LC/MS pos early & 1651 & $\underline{\mathrm{C} 00250}$ & $\underline{\mathrm{HMDB} 01545}$ & 1050 & 1.10 & 0.94 & 1.16 \\
\hline 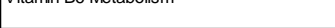 & pyridoxate & LC/MS neg & 31555 & $\underline{\mathrm{C} 00847}$ & HMDB00017 & 6723 & 1.48 & 0.96 & 1.55 \\
\hline Benzoate Motaholism & 4-hydroxyhippurate & LC/MS neg & 35527 & & $\underline{\mathrm{HMDB} 13678}$ & 151012 & 0.34 & 1.36 & 0.25 \\
\hline & 4-hydroxybenzoate & LC/MS neg & 21133 & $\underline{\mathrm{C} 00156}$ & HMDB00500 & 135 & 0.69 & 1.06 & 0.65 \\
\hline & 2,3-dihydroxyisovalerate & LC/MS polar & 38276 & C04039 & HMDB12141 & 677 & 0.39 & 1.13 & 0.35 \\
\hline & 2-isopropylmalate & LC/MS polar & 15667 & $\underline{\mathrm{C} 02504}$ & HMDB00402 & 77 & 0.87 & 0.84 & 1.04 \\
\hline & gluconate & LC/MS polar & 587 & $\mathrm{C} 00257$ & HMDB00625 & 10690 & 0.88 & 0.87 & 1.01 \\
\hline & ergothioneine & LC/MS pos early & 37459 & $\underline{\mathrm{C} 05570}$ & HMDB03045 & 3032311 & 1.10 & 1.17 & 0.94 \\
\hline & erythritol & LC/MS polar & 20699 & $\mathrm{C} 00503$ & HMDB02994 & 222285 & 0.76 & 1.03 & 0.74 \\
\hline Food Component/Plant & kojibiose & LC/MS polar & 21040 & C19632 & HMDB11742 & 164939 & 1.20 & 1.14 & 1.06 \\
\hline & panose & LC/MS polar & 37284 & $\underline{\mathrm{C} 00713}$ & HMDB11729 & 5288421 & 1.23 & 0.87 & 1.42 \\
\hline & quinate & LC/MS polar & 18335 & $\underline{\mathrm{C} 00296}$ & HMDB03072 & 6508 & 0.80 & 1.03 & 0.78 \\
\hline & stachydrine & LC/MS pos early & 34384 & $\underline{\mathrm{C} 10172}$ & HMDB04827 & 115244 & 0.52 & 0.55 & 0.95 \\
\hline & methyl glucopyranoside (alpha + beta) & LC/MS pos early & 46144 & & & & 1.10 & 0.98 & 1.13 \\
\hline & 2-keto-3-deoxy-gluconate & LC/MS polar & 48141 & $\underline{\mathrm{C} 00204}$ & HMDB01353 & 161227 & 0.68 & 0.43 & 1.58 \\
\hline Drug - Topical Agents & salicylate & LC/MS polar & 1515 & $\underline{\mathrm{C} 00805}$ & HMDB01895 & 338 & 0.59 & 0.67 & 0.88 \\
\hline Chemical & succinimide & LC/MS polar & 41888 & $\underline{\mathrm{C} 07273}$ & & 11439 & 1.26 & 1.11 & 1.14 \\
\hline Chemical & thioproline & LC/MS pos early & 53231 & & & 93176 & 0.77 & 1.00 & 0.77 \\
\hline
\end{tabular}

Table S1: Metabolomics of Repo>20x Shibire fly heads

All measured metabolites and their respective categories are listed for samples from Repo-GAL4>UAS-20xShibire, and both parental controls. Welch's t-test was performed on scaled signal for each metabolite, comparing each condition. Green highlighting marks a 
bioRxiv preprint doi: https://doi.org/10.1101/2022.02.17.480875; this version posted February 21, 2022. The copyright holder for this preprint (which was not certified by peer review) is the author/funder, who has granted bioRxiv a license to display the preprint in perpetuity. It is made available under aCC-BY-NC-ND 4.0 International license.

significant difference $(p \leq 0.05)$ between the groups, where metabolite ratio is $<1.00$, while light green is not significant, but close to the threshold $(0.05<p<0.10)$. Red highlighting marks a significant difference $(p \leq 0.05)$ between groups where metabolite ratio is $\geq 1.00$, and light red is not significant, but close to the threshold $(0.05<\mathrm{p}<0.10)$.

A.

A.

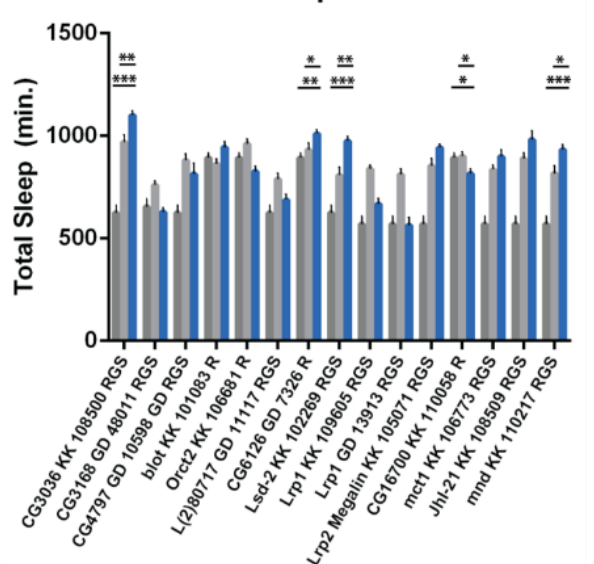

C.

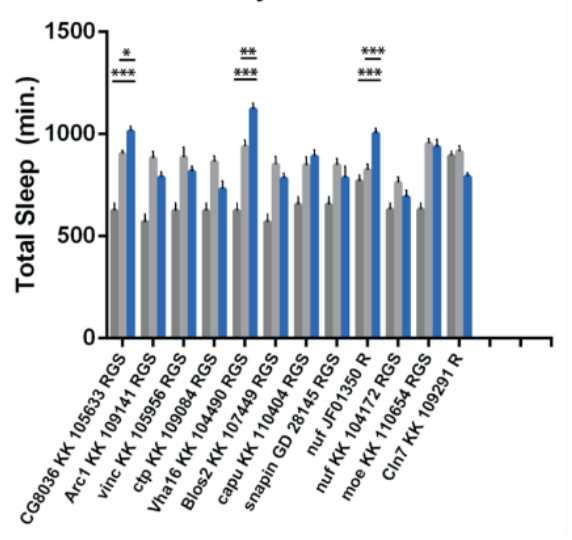

Transporters 2

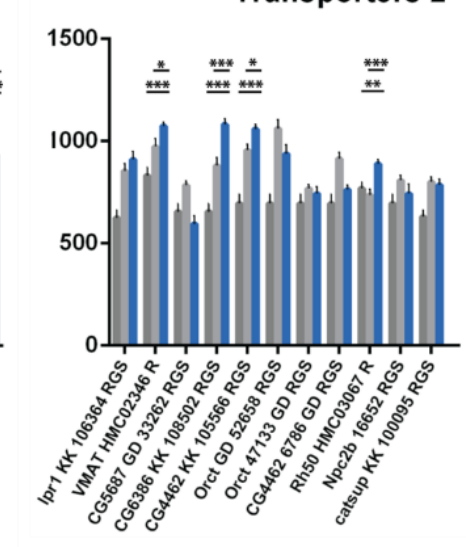

D.

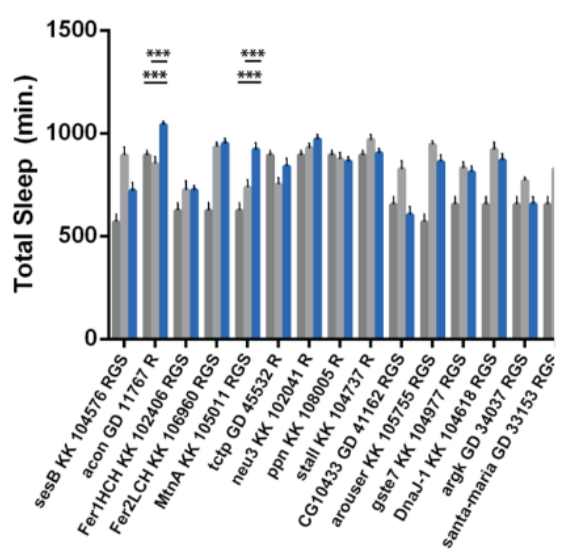

B.

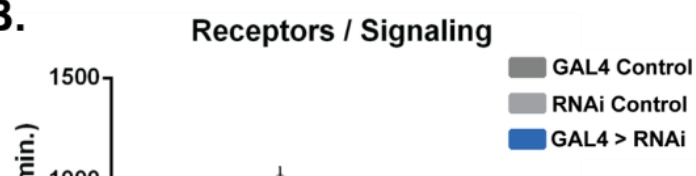

Figure 2 supplement 1: Pan-glial RNAi knockdown screen of candidate genes enriched in the barrier glia

Total sleep in female flies with RNAi knockdown of listed genes (KK and GD are VDRC collections, TRiP lines are from Bloomington Stock Center) (A)Transporters, (B) Receptors/Signaling pathway factors, (C) Cytoskeleton/Trafficking factors and (D) other genes by either Repo-Gal4 (labeled R) or UAS-Dicer; RepoGeneSwitch on RU+ food (labeled RGS). $\mathrm{n}=9$ - 16 flies per genotype, median = 16 . One-way ANOVA, with Holk-Sidak post-hoc comparisons. ${ }^{*} p<0.05,{ }^{* *} p<0.01,{ }^{* * *} p<0.001$. Error bars represent standard error of the mean (SEM). Significance values only marked for genes in which experimental flies were different from both parental controls. Certain experiments were performed simultaneously and therefore share a Gal4 control, re-plotted per each gene. 
bioRxiv preprint doi: https://doi.org/10.1101/2022.02.17.480875; this version posted February 21, 2022. The copyright holder for this preprint (which was not certified by peer review) is the author/funder, who has granted bioRxiv a license to display the preprint in perpetuity. It is made available under aCC-BY-NC-ND 4.0 International license.

A

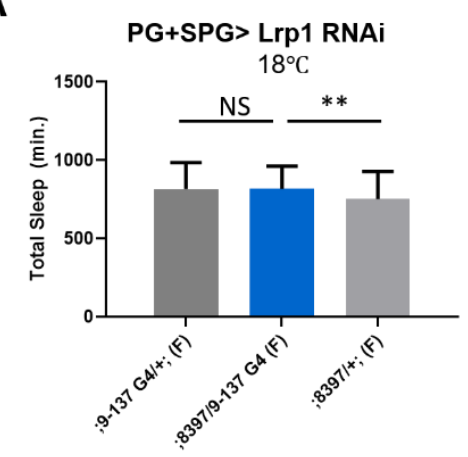

C

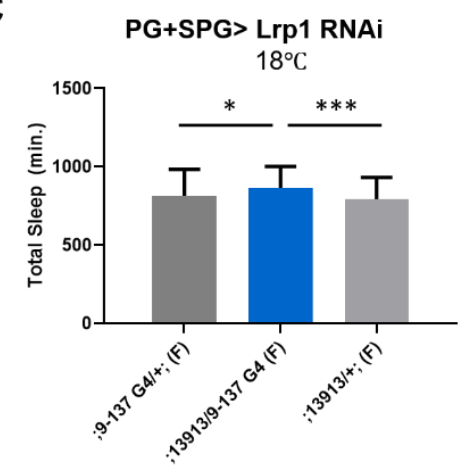

E

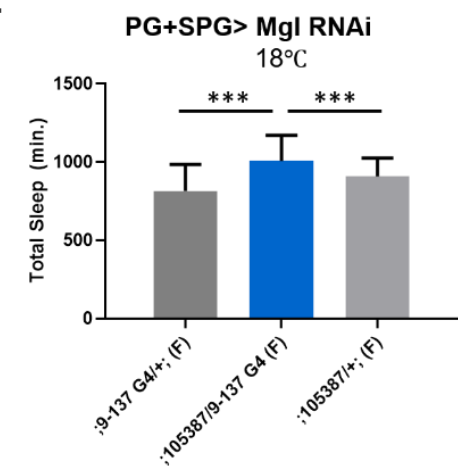

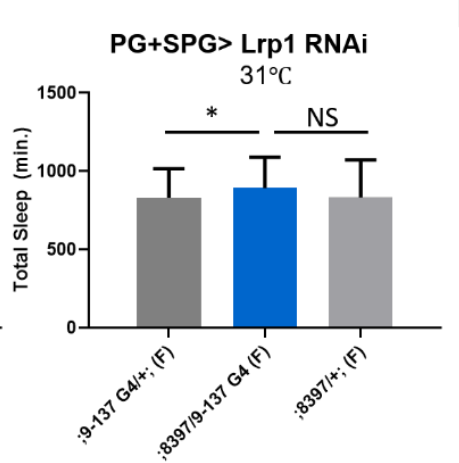
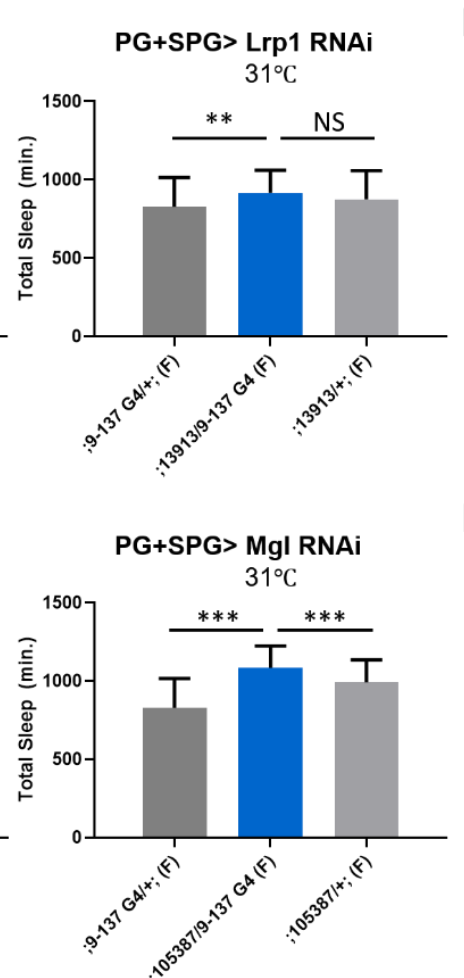

B

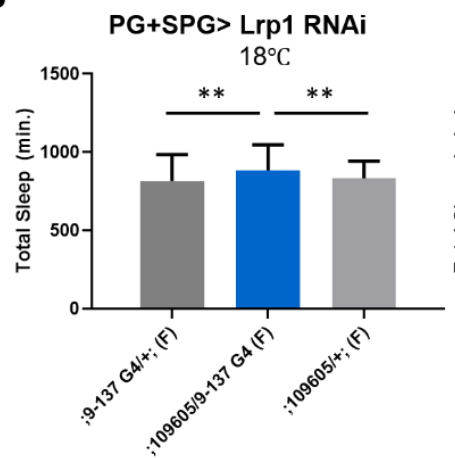

D

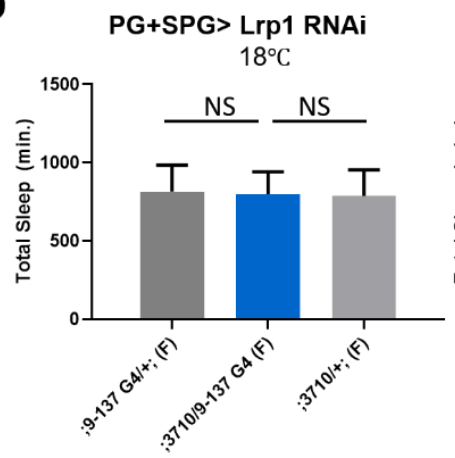

$\mathbf{F}$

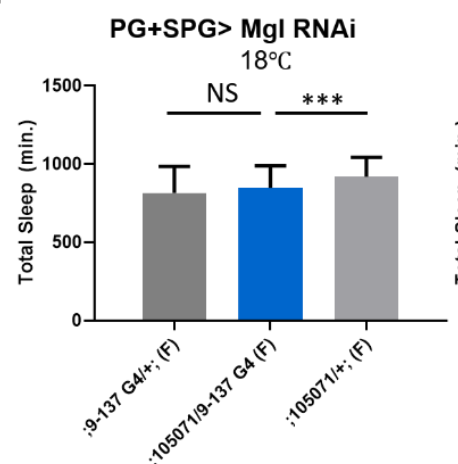

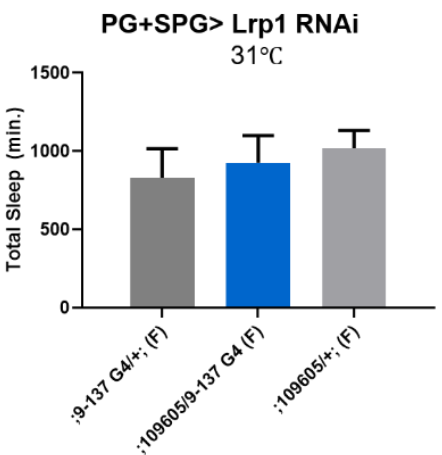

PG+SPG> Lrp1 RNAi

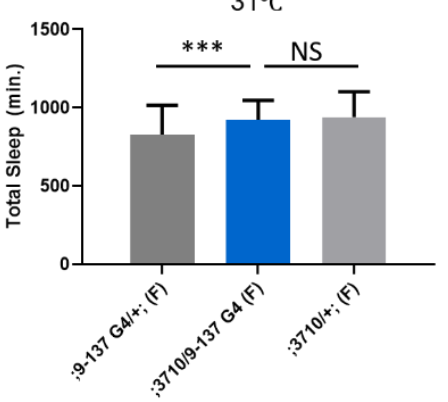

PG+SPG> MgI RNAi

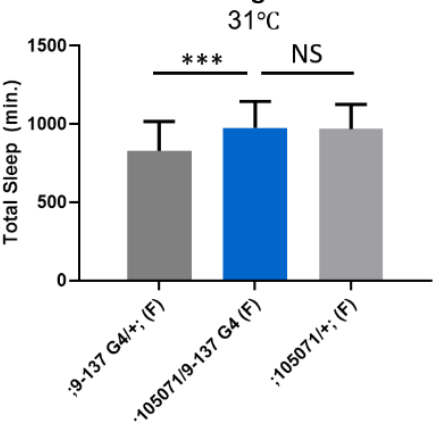

Figure 3 supplement 1 . Sleep time changes with knockdown of Lrp genes in surface glia

Total sleep in female flies with knockdown of (A) Lrp1 (8397 GD) (B)Lrp1(109605 KK) (C) Lrp1 (13913 KK) (D) Lrp1 (3710 GD) (E) Megalin (105387 KK) (F) Megalin (105071 KK) driven by (PG+SPG) driver 9-137-GAL4, $n=40-48$ per genotype at $18^{\circ} \mathrm{C}$ (permissive) and at $31{ }^{\circ} \mathrm{C}$ (restrictive). One-way ANOVA, with Holk-Sidak post-hoc comparisons. ${ }^{*} p<0.05,{ }^{* *} p<0.01,{ }^{* * *} p<0.001$. Error bars represent standard error of the mean (SEM). 